\title{
Low Dimensional MIMO Systems with Finite Sized Constellation Inputs
}

\author{
Rizwan Ghaffar and Raymond Knopp \\ Eurecom \\ FRANCE
}

\section{Introduction}

The seminal works in (Foschini \& Gans, 1998) and (Telatar, 1999) on multiple antenna elements at the transmitter and the receiver show a huge increase in the throughput of this pointto-point channel referred to also as multiple input multiple output (MIMO) system. These promising results of high spectral efficiency and enhanced reliability shifted the focus of research on multi antenna communications and motivated the introduction of multiple antenna elements in the future communication systems. Researchers persist to strive for finding space time codes (STC) with reduced decoding complexity. These codes take into account both the spatial and temporal dimensions of the MIMO channel. Orthogonal Space-Time Block Codes (OSTBCs) (Larsson \& Stoica, 2003) are widely used because they are easy to encode and decode. For the case of two transmit antennas, the OSTBC is known as Alamouti code (Alamouti, 1998). OSTBCs are repetition codes that only provide diversity gain. In order to approach the capacity limit they have to be used in concatenation with an outer code. Remarkable coding gains can be obtained if a capacity achieving temporal encoder, such as turbo or Low-Density Parity Check (LDPC) code is used in concatenation with a STC (Gonzalez-Lopez et al., 2006). Recently it has been shown for the ergodic channels that the complex concatenation of the STC and the outer codes can be replaced with temporally coded and spatially multiplexed streams (coded spatial streams) for nearing capacity (Ghaffar \& Knopp, 2008a). Each spatial stream can also be independently coded using temporal encoders as convolutional, turbo or LDPC codes whereas at the receiver, standard off-the-shelf decoders are used after the demodulator. To combat the frequency selectivity of MIMO wireless channels with low complexity equalization at the receivers, MIMO OFDM is the appropriate alternative. To contest the inherent fading of MIMO OFDM wireless channels, improved code diversity of bit interleaved coded modulation (BICM) for fading channels is rendering it the preferred option. Consequently the future wireless systems shall be based on BICM MIMO OFDM systems. However the requisite antenna spacing combined with the complexity constraints at the receiver are restricting the future MIMO based communication systems to the maximum of 4 spatial streams whereas it is reduced to 2 spatial streams in most scenarios. The existing and forthcoming standards as IEEE 802.11n (802.11n, 2006), IEEE 802.16m (802.16m, 2007) and Third Generation Partnership Project Long Term Evolution (3GPP LTE) (LTE, 2006) substantiate this argument.

This chapter therefore focuses on low dimensional spatially multiplexed time coded BICM MIMO OFDM systems with first part being devoted to the transmission strategies and corresponding receiver structures for such systems in the broadcast scenario while second part 
deliberates on interference suppression in such systems in the cellular scenario. This chapter particularly takes into account the finite sized constellation inputs and departs from the customary idealistic Gaussian assumption for the codewords. Each part is also accompanied by relevant information theoretic analysis and by simulation results under the settings of upcoming wireless standards.

\section{MIMO Broadcast scenario}

This part deliberates on the broadcast scenario of BICM MIMO OFDM system though the discussion also remains valid for the point-to-point MIMO systems. We consider the transmission strategy in which each spatial stream is independently encoded and modulated. We focus on the case of uniform power and nonuniform rate spatial streams (Ghaffar \& Knopp, 2008a) and the case of uniform rate and nonuniform power distribution (Ghaffar \& Knopp, 2008b) between these spatial streams. In such a broadcast scenario, receiver consequently views a multiple access channel (MAC). Shamai (Shamai \& Steiner, 2003) termed the approach of single code layer at each transmit antenna as MAC-outage approach. The reception is consequently based on successive interference cancellation (SIC) i.e. sequential decoding and subtraction (stripping) of spatial streams which introduces unequal error protection (UEP). This can be coarsely regarded as MMSE DFE as described in (Varanasi \& Guess, 1997). The idea of multiple data streams with UEP adds flexibility to the system which can be exploited for having prioritized users or advanced services in MIMO broadcast systems and in multimedia broadcast multicast services (MBMS). For instance it can be the broadcast of multimedia streams with different rates (quality) of the same data and the users decoding the stream depending on the received SNR. It can also be the broadcast of low and high rate streams (as audio and video) with prioritized or high SNR users decoding both audio and video streams while low SNR users decoding only the low rate audio stream. It is also applicable to high-definition TV (HDTV) scenario where low priority/quality users are able to receive standard-definition TV (SDTV) transmission while high priority/quality users access HDTV. This idea has limited similarity to superposition codes (Liu et al., 2002) whose signal space has a cloud/satellite topology. Cloud centers because of relatively higher distance amongst them carry information for low quality receivers whereas better receivers having larger noise tolerance can resolve up to the actual transmitted satellite symbol within the cloud.

For coded spatial streams (also for the STC), the well-known data model after appropriate filtering and sampling is $\mathbf{y}=\mathbf{H x}+\mathbf{z}$ (to be made precise in the subsequent sections) where $\mathbf{y}$ is the received data, $\mathbf{H}$ is the channel matrix, $\mathbf{x}$ is the symbol vector with the elements from finite constellations and $\mathbf{z}$ is the noise. The problem is then to detect some or all elements of $\mathbf{x}$ from $\mathbf{y}$. Essentially the same problem occurs in multiuser detection for CDMA (Verdu, 1998) and for single-carrier transmission over channels that induce intersymbol interference. In these cases, the matrix $\mathbf{H}$ usually has a specific structure.

The problem of detection of $\mathbf{x}$ from $\mathbf{y}$ has stimulated a large body of research (Verdu, 1998) and references therein. One can easily show that if the noise $\mathbf{z}$ is Gaussian then obtaining the maximum-likelihood (ML) solution for some or all elements of $\mathbf{x}$ is equivalent to minimizing the Euclidean distance $\|\mathbf{y}-\mathbf{H x}\|^{2}$ with respect to $\mathbf{x}$ over the finite set spanned by all possible combinations of constellation points that can constitute the vector $\mathbf{x}$. For ML soft MIMO detection, the demodulator calculates the log-likelihood ratios (LLRs) for all bits that constitute the desired elements of $\mathbf{x}$ by summing the Euclidean distances for the values of $\mathbf{x}$ for which that particular bit of the desired element of $\mathbf{x}$ is one and zero thereby amounting to $2^{\log \left(M_{1}\right)+\cdots+\log \left(M_{n_{r}}\right)}$ terms where $M_{k}$ is the modulation alphabet of the $k$-th spatial stream and 
$n_{r}$ is the total number of spatial streams (Larsson \& Jalden, 2008). In many cases of practical interest, one resorts to the approximation of replacing the sums with the largest term which is equivalent to minimizing the Euclidean distance and is termed as max log MAP approach. Unfortunately this problem is NP-hard for general $\mathbf{H}$ and $\mathbf{y}$ (Verdu, 1989) which implies that there are no known efficient (i.e. polynomial-time) solutions. Many sophisticated methods as lattice reduction and sphere decoding (Hochwald \& Brink, 2003) exist which find the ML solution with high probability, but these methods are in general still computationally complex. This is true also in an average sense if $\mathbf{H}$ is random (i.e. for a fading channel). The popular "sphere decoding" method is much more efficient than a brute-force search, but it still admits an average complexity that is exponential in the dimension of $\mathbf{x}$.

Naive solutions, like neglecting the integer constraint coupled with the Gaussian assumption for the alphabets and then subsequently projecting the so-obtained solution onto the finite set of permissible $\mathbf{x}$ [linear receivers as LMMSE and zero forcing ZF)], in general work poorly especially at lower SNRs. Standard linear detection approaches are further based on ignoring the spatial color at the output of linear detectors which results in the decoupling of spatial streams thereby fundamentally reducing the complexity of detection. These disregards proliferate the suboptimality of linear receivers which exhibit degraded performance especially at lower SNRs.

Standard receiver solutions for spatially multiplexed broadcast schemes including V-BLAST (Wolniansky et al., 1998) (Golden et al., 1999) use stripping decoders which incorporate suboptimal linear minimum mean square error (MMSE) filters (Medvedev et al., 2006) against the yet undecoded streams at each successive cancellation stage. MMSE because of its relative improved performance in the family of linear detectors is the preferred choice. Its optimality for power constrained Gaussian alphabets is well known but it is suboptimal for finite size constellations. Gaussian assumption of the post detection interference is open to discussion. Its behavior is close to Gaussian under various asymptotic conditions which include large SNRs and large number of transmit and receive antennas (Poor \& Verdu, 1997). But the fidelity of Gaussian assumption in a low dimensional system at moderate SNRs is questionable. Degradation of the performance due to the suboptimality combined with the complexity in the calculation of linear equalizers at each frequency tone (in OFDM based system) renders their real-time implementation debatable especially in fast fading wideband environments.

\subsection{System Model}

Before deliberating further on these receiver structures, we discuss the system model. As the overall system is based on BICM MIMO OFDM, it is imperative to first understand the significance and the implication of using BICM.

\subsubsection{BICM SISO System}

BICM because of its improved code diversity for fading channels and its flexibility to variable transmission rates, is a likely choice for future wireless systems as IEEE 802.11n (802.11n, 2006), IEEE 802.16m (802.16m, 2007) and 3GPP LTE (LTE, 2006). The landmark paper of Caire (Caire et al., 1998) on BICM showed that on some channels, the separation of demodulation and decoding is beneficial, provided that the encoder output is interleaved bit wise and a suitable soft decision metric is used in the Viterbi decoder. Code diversity, and therefore the reliability of coded modulation over a Rayleigh channel, can be improved this way. The code diversity in this case is equal to the smallest number of distinct bits along any error event. This leads to a better coding gain over a fading channel when compared to other coded mod- 


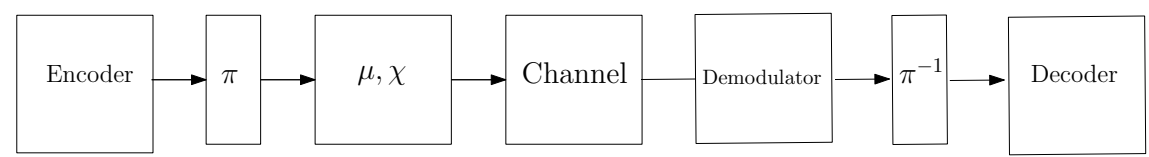

Fig. 1. Block Diagram of BICM system. $\pi$ denotes denotes a bit interleaver.

ulation schemes as Trellis Coded Modulation (TCM). BICM increases considerably Hamming distance while reducing (often marginally) Euclidean distance so BICM outperforms TCM over Rayleigh fading channel while suffering a moderate loss of performance over AWGN channel. If the channel model is nonstationary, in the sense that the propagation environment changes during transmission, then BICM provides a robust coding scheme.

The main idea of BICM is therefore to transform the channel generated by the multilevel constellation $\chi$ into parallel and independent binary channels. For transmission of complex modulation, channel is not binary but after bit interleaving, any transmission of a multilevel signal from $\chi$ with $|\chi|=2^{m}$, can actually be thought of as taking place over $m$ parallel channels, each carrying one binary symbol from the signal label. However, these channels are generally not independent, due to the constellation structure. To make them independent, binary symbols are interleaved over infinite length before being used as signal labels. The maximumlikelihood decoding (MLD) of BICM requires combined demodulation/decoding, which is often too complicated to implement. As a result, MLD is separated at the receiver, concatenating soft-metrics computation, deinterleaving and decoding. BICM block diagram is shown in fig. 1 which is the concatenation of an encoder for a code $C$ with an interleaver $\pi$ followed by a modulator $(\mu, \chi)$. In the decoder, the metrics reflect the fact of bits separation. Suppose that the code word to be transmitted is c. After interleaving and modulation, we transmit the codeword

$$
\mathbf{x}=\left(x_{1}, x_{2}, \ldots \ldots, x_{n}\right)
$$

and we receive $\mathbf{y}$ at the output of a stationary memoryless channel. With symbol interleaving, we decode by maximizing the metric

$$
\log p(\mathbf{y} \mid \mathbf{x})=\sum_{k=1}^{n} \log p\left(y_{k} \mid x_{k}\right)
$$

with respect to $\mathbf{x}$.

The bit interleaver can be seen as a one-to-one correspondence $\pi: k^{\prime} \rightarrow(k, i)$, where $k^{\prime}$ denotes the original ordering of the coded bits $c_{k^{\prime}}, k$ denotes the time ordering of the signals $x_{k}$ transmitted, and $i$ indicates the position of the bit $c_{k^{\prime}}$ in the symbol $x_{k}$. Let $\chi_{b}^{i}$ denote the subset of all signals $x \in \chi$ whose label has the value $b \in\{0,1\}$ in position $i$. Then the ML bit metric is given as

$$
\lambda^{i}\left(y_{k}, c_{k^{\prime}}\right)=\log \sum_{x \in \chi_{c_{k^{\prime}}}^{i}} p\left(y_{k} \mid x\right) \quad \text { where } \quad c_{k^{\prime}} \in[0,1] \quad \text { and } i=1,2, \ldots ., \log |\chi|
$$

So in case of BICM, it is the summation of bit metrics $\lambda^{i}\left(y_{k}, c_{k^{\prime}}\right)$ instead of the symbol metrics $\log p\left(y_{k} \mid x_{k}\right)$ for decoding. i.e.

$$
\underline{\hat{\mathbf{c}}}=\arg \max _{\underline{\mathbf{c}} \in C} \sum_{k^{\prime}} \lambda^{i}\left(y_{k}, c_{k^{\prime}}\right)
$$




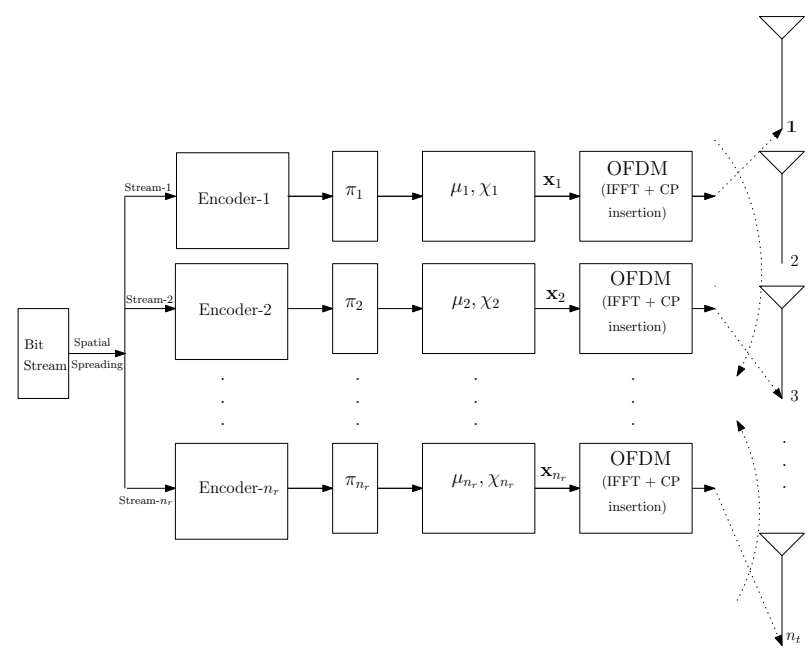

Fig. 2. Block diagram of Transmitter of $n_{t} \times n_{r}$ BICM MIMO OFDM system. $\pi_{1}$ denotes random interleaver, $\mu_{1}$ labeling map, $\chi_{1}$ signal set and $\mathbf{x}_{1}$ complex symbols vector for stream1.

The bit metrics (2) may be computationally too complex for implementation. Suboptimal simplified branch metric can be obtained by the $\log$-sum approximation $\log \sum_{j} z_{j} \approx \max _{j} \log z_{j}$. This yields

$$
\lambda^{i}\left(y_{k}, c_{k^{\prime}}\right)=\max _{x \in \chi_{c_{k^{\prime}}}^{i}} \log p\left(y_{k} \mid x\right)=\min _{\substack{x \in \chi_{c_{k^{\prime}}}^{i} \\ c_{k^{\prime}}}}\left|y_{k}-h_{k} x\right|^{2}
$$

where $h_{k}$ denotes the Rayleigh coefficient.

\subsubsection{BICM MIMO OFDM System}

We consider a MIMO broadcast system (without CSIT) which is a $n_{t} \times n_{r}\left(n_{t} \geq n_{r}\right)$ BICM MIMO OFDM system with $n_{r}$ spatial streams as shown in figs. 2 and 3 . We effectively reduce this to $n_{r} \times n_{r}$ system by antenna cycling at the transmitter (Foschini \& Gans, 1998) with each stream being transmitted by one antenna in any dimension. The antenna used by a particular stream is randomly assigned per dimension so that each stream sees all degrees of freedom of the channel. Let the spatial streams be $\underline{\mathbf{x}}_{1}, \cdots, \underline{\mathbf{x}}_{n_{r}} . x_{l}$ is the symbol of $\underline{\mathbf{x}}_{l}$ over a signal set $\chi_{l} \subseteq \mathcal{C}$ with a Gray labeling map $\mu_{l}:\{0,1\}^{\log _{2}\left|\chi_{l}\right|} \rightarrow \chi_{l}$. During the transmission of $l$-th spatial stream, the code sequence $\underline{\mathbf{c}}_{l}$ is interleaved by $\pi_{l}$ and then is mapped onto the signal sequence $\underline{\mathbf{x}}_{l} \in \chi_{l}$. Bit interleaver for the $l$-th stream can be modeled as $\pi_{l}: k^{\prime} \rightarrow(k, i)$ where $k^{\prime}$ denotes the original ordering of the coded bits $c_{k^{\prime}}$ of the $l$-th stream, $k$ denotes the time ordering of the signal $x_{l, k}$ and $i$ indicates the position of the bit $c_{k^{\prime}}$ in the symbol $x_{l, k}$.

We assume that the frequency reuse factor is one and cyclic prefix $(\mathrm{CP})$ of appropriate length is added to the OFDM symbols. Cascading IFFT at the transmitter and FFT at the receiver 


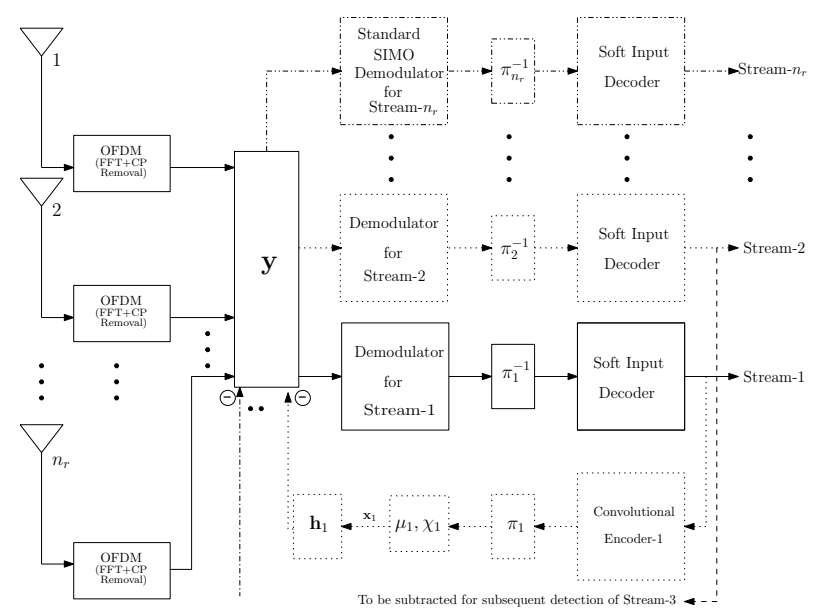

Fig. 3. Block diagram of SIC Receiver of BICM MIMO OFDM system. $\pi_{1}^{-1}$ denotes deinterleaver and $\mathbf{h}_{1}$ denotes the channel seen by stream- 1 .

with CP extension, transmission at the $k$-th frequency tone can be expressed as:-

$$
\begin{aligned}
\mathbf{y}_{k} & =\mathbf{h}_{1, k} x_{1}+\mathbf{h}_{2, k} x_{2}+\cdots+\mathbf{h}_{n_{r}, k} x_{n_{r}}+\mathbf{z}_{k}, \quad k=1,2, \cdots, T \\
& =\mathbf{H}_{k} \mathbf{x}_{k}+\mathbf{z}_{k}
\end{aligned}
$$

where $\mathbf{H}_{k}=\left[\mathbf{h}_{1, k} \cdots \mathbf{h}_{n_{r}, k}\right]$ i.e. the channel at the $k$-th frequency tone, $\mathbf{x}_{k}=\left[x_{1, k}, \cdots, x_{n_{r}, k}\right]^{T}$ and $(.)^{T}$ indicates the transpose operation. Each subcarrier corresponds to a symbol $\mathbf{x}$ from a constellation map $\chi_{1}, \cdots \chi_{n_{r}} \cdot \mathbf{y}_{k}, \mathbf{z}_{k} \in \mathbb{C}^{n_{r}}$ are the vectors of received symbols and circularly symmetric complex white Gaussian noise of double-sided power spectral density $N_{0} / 2$ at the $n_{r}$ receive antennas. $\mathbf{h}_{l, k} \in \mathbb{C}^{n_{r}}$ is the vector characterizing flat fading channel response from $l$-th transmitting antenna to $n_{r}$ receive antennas at $k$-th subcarrier. This vector has complexvalued multivariate Gaussian distribution with $E\left[\mathbf{h}_{l, k}\right]=\mathbf{0}$ and $E\left[\mathbf{h}_{l, k} \mathbf{h}_{l, k}^{+}\right]=\mathbf{I}$. The antennas at the transmitter are also assumed to be sufficiently spaced and therefore are uncorrelated. The complex symbols $x_{1, k}, \cdots, x_{n_{r}, k}$ of the spatial streams are assumed to be independent with variances $\sigma_{1}^{2}, \cdots, \sigma_{n_{r}}^{2}$ respectively. The channels at different subcarriers are also assumed to be independent. Bit metric for the bit $c_{k^{\prime}}$ at the $i$-th location of the symbol $x_{l, k}$ is given as

$$
\lambda_{l}^{i}\left(\mathbf{y}_{k^{\prime}}, c_{k^{\prime}}\right)=\log \sum_{x_{1} \in \chi_{1}} \cdots \sum_{x_{l} \in \chi_{l, c_{k^{\prime}}}^{i}} \cdots \sum_{x_{n_{r}} \in \chi_{n_{r}}} \exp \left[-\frac{1}{N_{0}}\left\|\mathbf{y}_{k}-\mathbf{H}_{k} \mathbf{x}\right\|^{2}\right]
$$

Applying log-sum approximation we have:-

$$
\lambda_{l}^{i}\left(\mathbf{y}_{k^{\prime}}, c_{k^{\prime}}\right) \approx \min _{x_{1} \in \chi_{1} \cdots x_{l} \in \chi_{l, c}^{i}, \cdots x_{k^{\prime}} \in \chi_{n_{r}}}\left[\left\|\mathbf{y}_{k}-\mathbf{H}_{k} \mathbf{x}\right\|^{2}\right]
$$




\subsection{Information Theoretic View}

We now calculate the mutual information of this system for the cases of Gaussian and finite sized constellation inputs.

\subsubsection{Gaussian Inputs}

The system equation ignoring the frequency index takes the form:-

$$
\mathbf{y}=\mathbf{h}_{1} x_{1}+\mathbf{h}_{2} x_{2}+\cdots+\mathbf{h}_{n_{r}} x_{n_{r}}+\mathbf{z}
$$

Since the receiver knows the realization of $\mathbf{H}$, the channel output is the pair $(\mathbf{y} ; \mathbf{H})=$ $(\mathbf{H x}+\mathbf{z} ; \mathbf{H})$. The mutual information between input and output is then Telatar (1999)

$$
\begin{aligned}
I(\mathbf{x} ;(\mathbf{y}, \mathbf{H})) & =I(\mathbf{x} ; \mathbf{H})+I(\mathbf{x} ; \mathbf{y} \mid \mathbf{H}) \\
& =I(\mathbf{x} ; \mathbf{y} \mid \mathbf{H}) \\
& =E_{\mathbf{H}} I(\mathbf{x} ; \mathbf{y} \mid \mathbf{H}=H)
\end{aligned}
$$

For the Gaussian inputs, we consider the following two cases:-

1. Spatial streams of uniform power and non-uniform rate.

2. Spatial streams of uniform rate and non-uniform power.

For Gaussian inputs, channel capacity of the system as per the chain rule (Foschini \& Gans, 1998) is

$$
I\left(x_{1}, x_{2} \cdots x_{n_{r}} ; \mathbf{y}\right)=I\left(x_{1} ; \mathbf{y}\right)+I\left(x_{2} ; \mathbf{y} \mid x_{1}\right)+\cdots+I\left(x_{n_{r}} ; \mathbf{y} \mid x_{1}, x_{2} \cdots x_{n_{r}-1}\right)
$$

The terms in the summation represent the channel capacities of each spatial stream once they are detected in the successive subtractive cancellation way. Conditioned on the channel, these terms can be written as:-

$$
\begin{gathered}
I\left(x_{1} ; \mathbf{y} \mid \mathbf{H}\right)=\log _{2}\left[\operatorname{det}\left\{\mathbf{I}+\sigma_{1}^{2} \mathbf{h}_{1} \mathbf{h}_{1}^{\dagger}\left(N_{0} \mathbf{I}+\sigma_{2}^{2} \mathbf{h}_{2} \mathbf{h}_{2}^{+}+\cdots+\sigma_{n_{r}}^{2} \mathbf{h}_{n_{r}} \mathbf{h}_{n_{r}}^{\dagger}\right)^{-1}\right\}\right] \\
I\left(x_{2} ; \mathbf{y} \mid \mathbf{H}, x_{1}\right)=\log _{2}\left[\operatorname{det}\left\{\mathbf{I}+\sigma_{2}^{2} \mathbf{h}_{2} \mathbf{h}_{2}^{\dagger}\left(N_{0} \mathbf{I}+\sigma_{3}^{2} \mathbf{h}_{3} \mathbf{h}_{3}^{\dagger}+\cdots+\sigma_{n_{r}}^{2} \mathbf{h}_{n_{r}} \mathbf{h}_{n_{r}}^{\dagger}\right)^{-1}\right\}\right]
\end{gathered}
$$

and

$$
I\left(x_{n_{r}} ; \mathbf{y} \mid \mathbf{H}, x_{1}, x_{2} \cdots, x_{n_{r}-1}\right)=\log _{2}\left(1+\frac{\sigma_{n_{r}}^{2}}{N_{0}}\left\|\mathbf{h}_{n_{r}}\right\|^{2}\right)
$$

where $\mathbf{H}=\left[\mathbf{h}_{1} \mathbf{h}_{2} \cdots \mathbf{h}_{n_{r}}\right]$ is the channel matrix. Fig. 4 shows the ergodic capacity for the case of $2 \times 2$ system with spatial streams of uniform power and nonuniform rate. Note that SNR is the received SNR per antenna i.e. SNR $=\frac{\sigma_{1}^{2}+\sigma_{2}^{2}}{N_{0}}$. It is evident that the stream to be detected first has lower capacity as compared to the stream to be detected last which enjoys higher diversity.

Fig. 5 compares two cases of spatial streams with uniform power and nonuniform rate and spatial streams with uniform rate and nonuniform power for $2 \times 2,3 \times 3$ and $4 \times 4$ systems. Key to the optimality of stripping is the use of Gaussian inputs as long as the stripping decoders incorporate MMSE filters against yet undecoded streams at each successive cancellation stage. Successive stripping requires that each stream must be transmitted at a different 
rate with uniform power. We investigate a slightly suboptimal solution where we guarantee equal rate with nonuniform powers on each stream. Numerical optimization revealed that uniform rate and nonuniform power distribution leads to negligible suboptimality as shown in fig. 5.

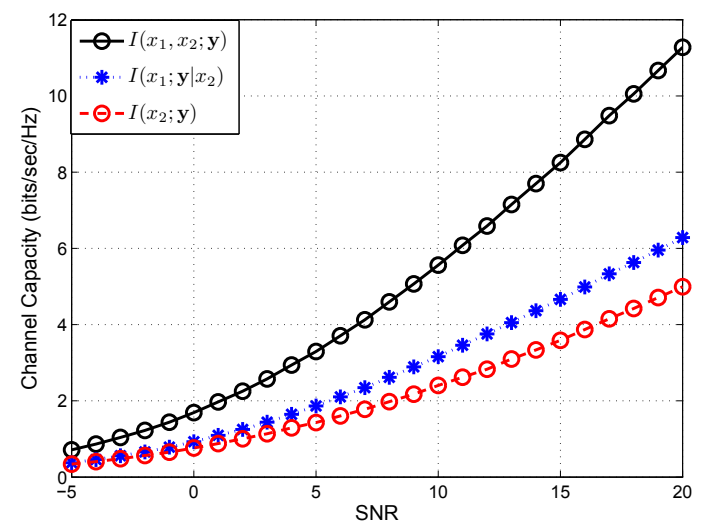

Fig. 4. Capacity of $2 \times 2$ system for Gaussian alphabets for the case of uniform power and nonuniform rate spatial streams.

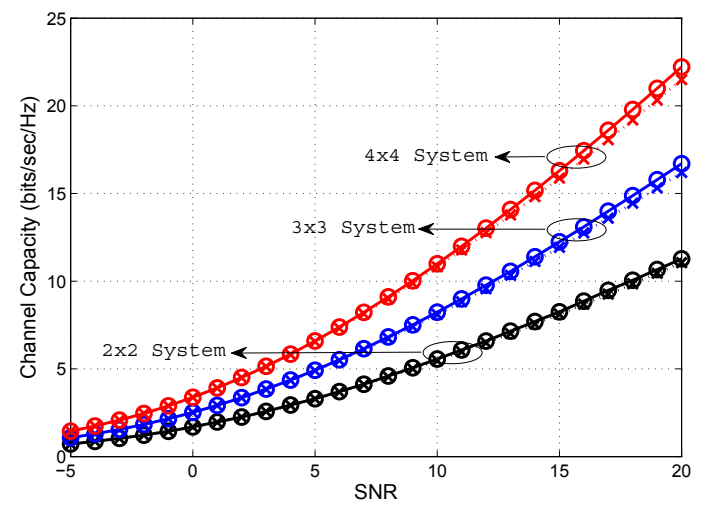

Fig. 5. Capacity of $2 \times 2,3 \times 3$ and $4 \times 4$ systems for Gaussian alphabets for the cases of spatial streams of uniform power and nonuniform rate and the spatial streams of uniform rate and nonuniform power. Note that the circles indicate the case of uniform power and nonuniform rate spatial streams while crosses indicate the case of uniform rate and nonuniform power spatial streams. 


\subsubsection{Finite Sized Constellation Inputs}

To reduce the complexity and enhance the understanding of mutual information for finite sized constellation inputs, we restrict to the case of dual stream transmission. The system equation ignoring the frequency index takes the form:-

$$
\mathbf{y}=\mathbf{h}_{1} x_{1}+\mathbf{h}_{2} x_{2}+\mathbf{z}
$$

Mutual information expression for the dual streams from the chain rule (Foschini \& Gans, 1998) is given as

$$
I\left(\mathbf{y} ; x_{1}, x_{2}\right)=I\left(\mathbf{y} ; x_{1}\right)+I\left(\mathbf{y} ; x_{2} \mid x_{1}\right)
$$

For equal power distribution, $I\left(\mathbf{y} ; x_{1}\right)<I\left(\mathbf{y} ; x_{2} \mid x_{1}\right)$ dictating rate of first stream being less than rate of second stream $\left(R_{1}<R_{2}\right)$. For finite size QAM constellation with $x_{1} \in M_{1}$ and $x_{2} \in$ $M_{2}$, the mutual information expression conditioned on the channel takes the form (Ghaffar \& Knopp, 2008a)

$$
\begin{aligned}
I\left(\mathbf{y} ; x_{1} \mid \mathbf{H}\right) & =\mathcal{H}\left(x_{1} \mid \mathbf{H}\right)-\mathcal{H}\left(x_{1} \mid \mathbf{y}, \mathbf{H}\right) \\
& =\log M_{1}-\mathcal{H}\left(x_{1} \mid \mathbf{y}, \mathbf{H}\right)
\end{aligned}
$$

where $\mathcal{H}()=.-E \log p($.$) is the entropy function. Second term of eq. (10) is given as:-$

$$
\begin{aligned}
\mathcal{H}\left(x_{1} \mid \mathbf{y}, \mathbf{H}\right) & =\sum_{x_{1}} \int_{\mathbf{y}} \int_{\mathbf{H}} p\left(x_{1}, \mathbf{y}, \mathbf{H}\right) \log \frac{1}{p\left(x_{1} \mid \mathbf{y}, \mathbf{H}\right)} d \mathbf{y} d \mathbf{H} \\
& =\sum_{x_{1}} \int_{\mathbf{y}} \int_{\mathbf{H}} p\left(x_{1}, \mathbf{y}, \mathbf{H}\right) \log \frac{p(\mathbf{y}, \mathbf{H})}{p\left(x_{1}, \mathbf{y}, \mathbf{H}\right)} d \mathbf{y} d \mathbf{H} \\
& =\sum_{x_{1}} \sum_{x_{2}} \int_{\mathbf{y}} \int_{\mathbf{H}} p\left(x_{1}, x_{2}, \mathbf{y}, \mathbf{H}\right) \log \frac{\sum_{x_{1}^{\prime}} \sum_{x_{2}^{\prime}} p\left(\mathbf{y} \mid x_{1}^{\prime}, x_{2}^{\prime}, \mathbf{H}\right)}{\sum_{x_{2}^{\prime}} p\left(\mathbf{y} \mid x_{1}, x_{2}^{\prime}, \mathbf{H}\right)} d \mathbf{y} d \mathbf{H}
\end{aligned}
$$

For our purposes, it suffices to note that for each choice of $x_{1}$ and $x_{2}$, there are two sources of randomness in the choices of channel and noise. The above quantities can be easily approximated numerically using sampling (Monte-Carlo) methods with $N_{\mathbf{z}}$ realizations of noise and $N_{\mathbf{H}}$ realizations of the channel i.e.

$$
\begin{aligned}
\mathcal{H}\left(x_{1} \mid \mathbf{y}, \mathbf{H}\right) & =\frac{1}{M_{1} M_{2} N_{\mathbf{z}} N_{\mathbf{H}}} \sum_{x_{1}} \sum_{x_{2}} \sum_{\mathbf{H}}^{N_{\mathbf{H}}} \sum_{\mathbf{z}}^{N_{\mathbf{z}}} \log \frac{\sum_{x_{1}^{\prime}} \sum_{x_{2}^{\prime}} \exp \left[-\frac{1}{N_{0}}\left\|\mathbf{y}-\mathbf{h}_{1} x_{1}^{\prime}-\mathbf{h}_{2} x_{2}^{\prime}\right\|^{2}\right]}{\sum_{x_{2}^{\prime}} \exp \left[-\frac{1}{N_{0}}\left\|\mathbf{y}-\mathbf{h}_{1} x_{1}-\mathbf{h}_{2} x_{2}^{\prime}\right\|^{2}\right]} \\
& =\frac{1}{M_{1} M_{2} N_{\mathbf{z}} N_{\mathbf{H}}} \sum_{x_{1}} \sum_{x_{2}} \sum_{\mathbf{H}}^{N_{\mathbf{H}}} \sum_{\mathbf{z}}^{N_{\mathbf{z}}} \log \frac{\sum_{x_{1}^{\prime}} \sum_{x_{2}^{\prime}} \exp \left[-\frac{1}{N_{0}}\left\|\mathbf{h}_{1} x_{1}+\mathbf{h}_{2} x_{2}+\mathbf{z}-\mathbf{h}_{1} x_{1}^{\prime}-\mathbf{h}_{2} x_{2}^{\prime}\right\|^{2}\right]}{\sum_{x_{2}^{\prime}} \exp \left[-\frac{1}{N_{0}}\left\|\mathbf{h}_{2} x_{2}+\mathbf{z}-\mathbf{h}_{2} x_{2}^{\prime}\right\|^{2}\right]}
\end{aligned}
$$


Similarly the mutual information of second stream conditioned on the channel when first stream has been detected is given by:-

$$
\begin{aligned}
I\left(\mathbf{y} ; x_{2} \mid x_{1}, \mathbf{H}\right) & =\mathcal{H}\left(x_{2} \mid x_{1}, \mathbf{H}\right)-\mathcal{H}\left(x_{2} \mid \mathbf{y}, x_{1}, \mathbf{H}\right) \\
& =\log M_{2}-\sum_{x_{1}} \sum_{x_{2}} \int_{\mathbf{y}} \int_{\mathbf{H}} p\left(x_{1}, x_{2}, \mathbf{y}, \mathbf{H}\right) \log \frac{1}{p\left(x_{2} \mid \mathbf{y}, x_{1}, \mathbf{H}\right)} d \mathbf{y} d \mathbf{H} \\
& =\log M_{2}-\sum_{x_{1}} \sum_{x_{2}} \int_{\mathbf{y}} \int_{\mathbf{H}} p\left(x_{1}, x_{2}, \mathbf{y}, \mathbf{H}\right) \log \frac{p\left(\mathbf{y}, x_{1}, \mathbf{H}\right)}{p\left(x_{1}, x_{2}, \mathbf{y}, \mathbf{H}\right)} d \mathbf{y} d \mathbf{H} \\
& =\log M_{2}-\sum_{x_{1}} \sum_{x_{2}} \int_{\mathbf{y}} \int_{\mathbf{H}} p\left(x_{1}, x_{2}, \mathbf{y}, \mathbf{H}\right) \log \frac{\sum_{x_{2}^{\prime}} p\left(\mathbf{y} \mid x_{1}, x_{2}^{\prime}, \mathbf{H}\right)}{p\left(\mathbf{y} \mid x_{1}, x_{2}, \mathbf{H}\right)} d \mathbf{y} d \mathbf{H}
\end{aligned}
$$

Estimation of this quantity using Monte-Carlo simulation

$$
\begin{aligned}
I\left(\mathbf{y} ; x_{2} \mid x_{1}, \mathbf{H}\right) & =\log M_{2}-\frac{1}{M_{1} M_{2} N_{\mathbf{z}} N_{\mathbf{H}}} \sum_{x_{1}} \sum_{x_{2}} \sum_{\mathbf{H}}^{N_{\mathbf{H}}} \sum_{\mathbf{z}}^{N_{\mathbf{z}}} \log \frac{\sum_{x_{2}^{\prime}} \exp \left[-\frac{1}{N_{0}}\left\|\mathbf{y}-\mathbf{h}_{1} x_{1}-\mathbf{h}_{2} x_{2}^{\prime}\right\|^{2}\right]}{\exp \left[-\frac{1}{N_{0}}\left\|\mathbf{y}-\mathbf{h}_{1} x_{1}-\mathbf{h}_{2} x_{2}\right\|^{2}\right]} \\
& =\log M_{2}-\frac{1}{M_{1} M_{2} N_{\mathbf{z}} N_{\mathbf{H}}} \sum_{x_{1}} \sum_{x_{2}} \sum_{\mathbf{H}}^{N_{\mathbf{H}}} \sum_{\mathbf{z}}^{N_{\mathbf{z}}} \log \frac{\sum_{x_{2}^{\prime}} \exp \left[-\frac{1}{N_{0}}\left\|\mathbf{h}_{2} x_{2}+\mathbf{z}-\mathbf{h}_{2} x_{2}^{\prime}\right\|^{2}\right]}{\exp \left[-\frac{1}{N_{0}}\|\mathbf{z}\|^{2}\right]}
\end{aligned}
$$

Fig. 6 shows the capacity of first stream once second stream is not yet decoded for different combinations of finite constellation alphabets. For moderate values of SNR, the capacity of first stream is a function of the yet undetected second stream and this capacity decreases as the rate (constellation size) of second stream increases. This degradation is not observed at low and high values of SNR as at low SNR, two streams are orthogonal while at high SNR, second stream can be perfectly stripped off leading to detection of first stream. Rate of first stream being a function of the rate of second stream leads to nonuniform rates in uniform power dual stream scenario and this leads to the following proposed broadcast strategy.

\subsection{Broadcast Strategy}

We restrict to dual stream scenario for the broadcast case. The broadcast approach in dual stream scenario based on UEP (MAC-outage (Shamai \& Steiner, 2003)) is motivated by the capacity of a Gaussian broadcast channel with two users i.e.

$$
\mathcal{C}=I\left(x_{1} ; y_{1}\right)+I\left(x_{2} ; y_{2} \mid x_{1}\right)
$$

where user 2 sees a better channel and so is able to decode and strip off the interference. The broadcast strategy (Ghaffar \& Knopp, 2008a) incorporates the transmission of two spatial streams of uniform power and nonuniform rate and incorporates two levels of performance. The reliably decoded information rate depends on the state of the channel which is determined by monitoring the received SNR being above or below a certain threshold. Transmitter is operating at a constant power and data rate but the limited adaptability of the system helps receivers to gear up to a higher data rate as the channel conditions improve. 


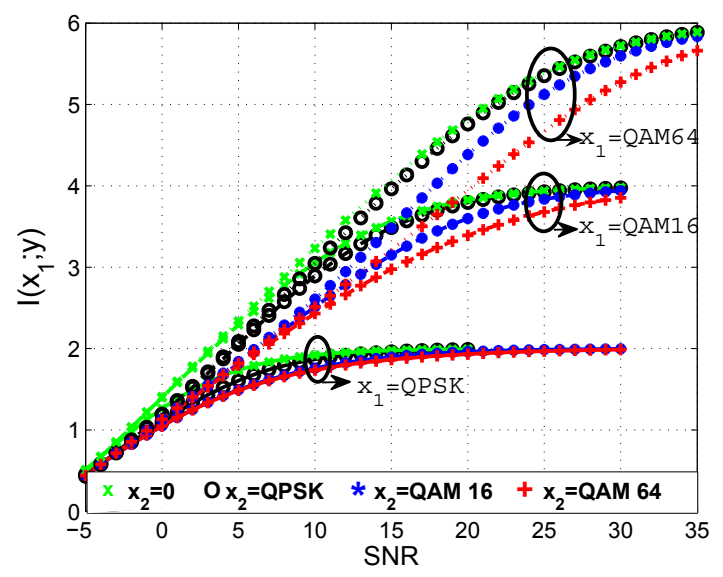

Fig. 6. Capacity of first stream in dual-stream broadcast approach for finite size alphabets once second stream is not known. Both streams have equal power. $\mathbf{x}_{2}=0$ indicates the special case when second stream has been decoded and stripped off. Note that SNR includes power of both streams.

Low priority/quality users are able to decode low rate stream $\mathbf{x}_{1}$ while high priority/quality users are able to decode both low and high rate streams i.e. $\mathbf{x}_{1}$ and $\mathbf{x}_{2}$ by successive stripping. The rates of two streams are

$$
R_{1} \leq I\left(\mathbf{y} ; x_{1}\right)
$$

and

$$
R_{2} \leq I\left(\mathbf{y} ; x_{2} \mid x_{1}\right)
$$

The notion of priority/quality is typically the received SNR and/or stream decoupling. The users are divided into two groups i.e. near-in users and far-out users based on their received $\mathrm{SNR}$. The lower rate stream $\mathbf{x}_{1}$ is designed for a lower value of $\mathrm{SNR}$ i.e. $\mathrm{SNR}_{1}$ while the higher rate stream $\mathbf{x}_{2}$ is designed for higher value of $\mathrm{SNR}$ i.e. $\mathrm{SNR}_{2}$. The received $\mathrm{SNR}$ of a particular user dictates two decoding options.

1. If $\mathrm{SNR}_{2}>\mathrm{SNR} \geq \mathrm{SNR}$, the user decodes $\mathbf{x}_{1}$.

2. If $\mathrm{SNR} \geq \mathrm{SNR}_{2}$, the user decodes both streams i.e. $\mathbf{x}_{1}$ and $\mathbf{x}_{2}$. The user first decodes low rate stream $\mathbf{x}_{1}$, strips it out and then decodes high rate stream $\mathbf{x}_{2}$.

This leads us to SIC detection based MIMO broadcast scenario with uniform power and nonuniform rate spatial streams. We now discuss the detectors for such broadcast scenario.

\subsection{Detectors}

The detectors discussed in this section are valid not only for spatially multiplexed MIMO systems but may be extended to other types of STC systems. We discuss two types of detectors as MMSE detector and low complexity max log MAP detector (Ghaffar \& Knopp, 2009b). 


\subsubsection{MMSE}

The frequency domain MMSE filter for $x_{1, k}$ is given as

$$
\mathbf{h}_{1, k}^{M M S E}=\left(\mathbf{h}_{1, k}^{\dagger} \mathbf{R}_{1, k}^{-1} \mathbf{h}_{1, k}+\sigma_{1}^{-2}\right)^{-1} \mathbf{h}_{1, k}^{\dagger} \mathbf{R}_{1, k}^{-1}
$$

where $\mathbf{R}_{1, k}=\sigma_{2}^{2} \mathbf{h}_{2, k} \mathbf{h}_{2, k}^{\dagger}+\sigma_{3}^{2} \mathbf{h}_{3, k} \mathbf{h}_{3, k}^{\dagger}+\cdots+\sigma_{n_{r}}^{2} \mathbf{h}_{n_{r}, k} \mathbf{h}_{n, k}^{\dagger}+N_{0} \mathbf{I}$. After the application of MMSE filter we get

$$
y_{k}=\alpha_{k} x_{1, k}+z_{k}
$$

where $z_{k}$ is assumed to be zero mean complex Gaussian random variable with variance $N_{k}=\mathbf{h}_{1, k}^{M M S E} \mathbf{R}_{1, k} \mathbf{h}_{1, k}^{M M S E^{\dagger}}$ and $\alpha_{k}=\mathbf{h}_{1, k}^{M M S E} \mathbf{h}_{1, k}$. Gaussianity has been assumed for post detection interference which increases the suboptimality of MMSE in the case of less number of interferers. Bit metric for the bit $c_{k^{\prime}}$ on first stream is given as:-

$$
\lambda_{1}^{i}\left(\mathbf{y}_{k}, c_{k^{\prime}}\right) \approx \min _{x_{1} \in \chi_{1, c_{k^{\prime}}}^{i}}\left[\frac{1}{N_{k}}\left|y_{k}-\alpha_{k} x_{1}\right|^{2}\right]
$$

where $\chi_{1, c_{k^{\prime}}}^{i}$ denotes the subset of the signal set $x_{1} \in \chi_{1}$ whose labels have the value $c_{k^{\prime}} \in$ $\{0,1\}$ in the position $i$. This metric has computational complexity $\mathcal{O}\left(\left|\chi_{1}\right|\right)$.

\subsubsection{Low complexity max log MAP Detector}

The max log MAP bit metric as per (6) is given as

$$
\lambda_{1}^{i}\left(\mathbf{y}_{k^{\prime}}, c_{k^{\prime}}\right) \approx \min _{x_{1} \in \chi_{1, c_{k^{\prime}}}^{i}, x_{2} \in \chi_{2}, \cdots, x_{n_{r}} \in \chi_{n_{r}}}\left\|\mathbf{y}_{k}-\mathbf{h}_{1, k} x_{1}-\cdots-\mathbf{h}_{n_{r}, k} x_{n_{r}}\right\|^{2}
$$

which has computational complexity $\mathcal{O}\left(\left|\chi_{1}\right| \cdots\left|\chi_{n_{r}}\right|\right)$. For brevity we drop the frequency index $k$ and the bit position index $k^{\prime}$ i.e.

$$
\begin{aligned}
& \lambda_{1}^{i}(\mathbf{y}, c) \approx \min _{x_{1} \in \chi_{1, c}^{i}, x_{2} \in \chi_{2}, \cdots, x_{n_{r}} \in \chi_{n_{r}}}\left\|\mathbf{y}-\mathbf{h}_{1} x_{1}-\cdots-\mathbf{h}_{n_{r}} x_{n_{r}}\right\|^{2} \\
&=\min _{x_{1} \in \chi_{1, c}^{i}, x_{2} \in \chi_{2}, \cdots, x_{n_{r}} \in \chi_{n_{r}}}\left\{\|\mathbf{y}\|^{2}+\sum_{j=1}^{n_{r}}\left\|\mathbf{h}_{j} x_{j}\right\|^{2}+2 \Re \sum_{j=1}^{n_{r}-1} \sum_{l=j+1}^{n_{r}}\left(\mathbf{h}_{j} x_{j}\right)^{\dagger}\left(\mathbf{h}_{l} x_{l}\right)-2 \Re \sum_{j=1}^{n_{r}}\left(\mathbf{h}_{j}^{\dagger} \mathbf{y}\right) x_{j}^{*}\right\} \\
&=\min _{x_{1} \in \chi_{1, c}^{i}, x_{2} \in \chi_{2}, \cdots, x_{n_{r}} \in \chi_{n_{r}}}\left\{\|\mathbf{y}\|^{2}+\sum_{j=1}^{n_{r}-1}\left\|\mathbf{h}_{j} x_{j}\right\|^{2}+2 \Re \sum_{j=1}^{n_{r}-1} \sum_{l=j+1}^{n_{r}-1} p_{j l} x_{j}^{*} x_{l}-2 \Re \sum_{j=1}^{n_{r}-1} y_{j} x_{j}^{*}\right. \\
&\left.+2 \Re \sum_{j=1}^{n_{r}-1} p_{j n_{r}} x_{j}^{*} x_{n_{r}}-2 \Re y_{n_{r}} x_{n_{r}}^{*}+\left\|\mathbf{h}_{n_{r}} x_{n_{r}}\right\|^{2}\right\}
\end{aligned}
$$

where $y_{k}=\mathbf{h}_{k}^{\dagger} \mathbf{y}$ be the matched filter (MF) output for $k$-th stream and $p_{k m}=\mathbf{h}_{k}^{\dagger} \mathbf{h}_{m}$ be the cross correlation between $k$-th and $m$-th channel. Breaking some of the terms in their real and 
imaginary parts with subscripts $(.)_{R}$ and $(.)_{I}$ indicating real and imaginary parts of a complex number, we have

$$
\begin{aligned}
\lambda_{1}^{i}(\mathbf{y}, c)= & \min _{x_{1} \in \chi_{1, c}^{i} \cdots x_{n_{r}} \in \chi_{n_{r}}}\left\{\sum_{j=1}^{n_{r}-1}\left\|\mathbf{h}_{j} x_{j}\right\|^{2}+2 \Re \sum_{j=1}^{n_{r}-1} \sum_{l=j+1}^{n_{r}-1} p_{j l} x_{j}^{*} x_{l}-2 \Re \sum_{j=1}^{n_{r}-1} y_{j} x_{j}^{*}\right. \\
& +\left(2 \sum_{j=1}^{n_{r}-1}\left(p_{j n_{r}, R} x_{j, R}+p_{j n_{r}, I} x_{j, I}\right)-2 y_{n_{r}, R}\right) x_{n_{r}, R}+\left\|\mathbf{h}_{n_{r}}\right\|^{2} x_{n_{r}, R}^{2} \\
& \left.+\left(2 \sum_{j=1}^{n_{r}-1}\left(p_{j n_{r}, R} x_{j, I}-p_{j n_{r}, I} x_{j, R}\right)-2 y_{n_{r}, I}\right) x_{n_{r}, I}+\left\|\mathbf{h}_{n_{r}}\right\|^{2} x_{n_{r}, I}^{2}\right\}
\end{aligned}
$$

This equation reduces one complex dimension of the system. For $x_{n_{r}}$ belonging to equal energy alphabets, the bit metric is written as

$$
\begin{aligned}
& \lambda_{1}^{i}(\mathbf{y}, c)=\min _{x_{1} \in \chi_{1, c}^{i} \cdots x_{n_{r}-1} \in \chi_{n_{r}-1}}\left\{\sum_{j=1}^{n_{r}-1}\left\|\mathbf{h}_{j} x_{j}\right\|^{2}+2 \Re \sum_{j=1}^{n_{r}-1} \sum_{l=j+1}^{n_{r}-1} p_{j l} x_{j}^{*} x_{l}-2 \Re \sum_{j=1}^{n_{r}-1} y_{j} x_{j}^{*}\right. \\
& \left.-\left|2 \sum_{j=1}^{n_{r}-1}\left(p_{j n_{r}, R} x_{j, R}+p_{j n_{r}, I} x_{j, I}\right)-2 y_{n_{r}, R}\right|\left|x_{n_{r}, R}\right|-\left|2 \sum_{j=1}^{n_{r}-1}\left(p_{j n_{r}, R} x_{j, I}-p_{j n_{r}, I} x_{j, R}\right)-2 y_{n_{r}, I}\right|\left|x_{n_{r}, I}\right|\right\}
\end{aligned}
$$

For $x_{n_{r}}$ belonging to non-equal energy alphabets, it's real and imaginary part which minimizes (23) are given as

$$
\begin{aligned}
& x_{n_{r}, R} \rightarrow-\frac{\sum_{j=1}^{n_{r}-1}\left(p_{j n_{r}, R} x_{j, R}+p_{j n_{r}, I} x_{j, I}\right)-y_{n_{r}, R}}{\left\|\mathbf{h}_{n_{r}}\right\|^{2}} \\
& x_{n_{r}, I} \rightarrow-\frac{\sum_{j=1}^{n_{r}-1}\left(p_{j n_{r}, R} x_{j, I}-p_{j n_{r}, I} x_{j, R}\right)-y_{n_{r}, I}}{\left\|\mathbf{h}_{n_{r}}\right\|^{2}}
\end{aligned}
$$

where $\rightarrow$ indicates the quantization process in which amongst the finite available points, the point closest to the calculated continuous value is selected.

This bit metric implies reduction in the complexity to $\mathcal{O}\left(\left|\chi_{1}\right| \cdots\left|\chi_{n_{r}-1}\right|\right)$. Reduction of one complex dimension without any additional processing is a fundamental result of significant importance for lower dimensional systems. Additionally this bit metric is based on MF outputs and channel correlations and is therefore simpler for fixed point implementations. The intricacy in the practical implementation of a higher dimensional MIMO system due to space (requisite antenna spacing) and technology constraints underlines the significance of complexity reduction algorithms for lower dimensional systems. MMSE based demodulators involve computationally complex operations of matrix inversions which are very hard for fixed point implementations. Moreover MMSE demodulator additionally needs the knowledge of noise variance. 


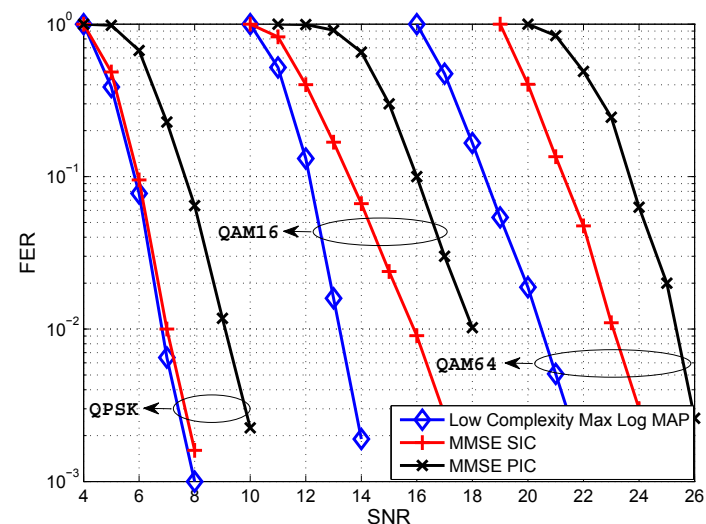

Fig. 7. $2 \times 2$ system with uniform rate and nonuniform power spatial streams. For QPSK $\sigma_{1}^{2}=0.63 P_{T}, \sigma_{2}^{2}=0.37 P_{T}$, for QAM $16 \sigma_{1}^{2}=0.67 P_{T}, \sigma_{2}^{2}=0.33 P_{T}$ while for QAM64 $\sigma_{1}^{2}=$ $0.70 P_{T}, \sigma_{2}^{2}=0.30 P_{T}$.

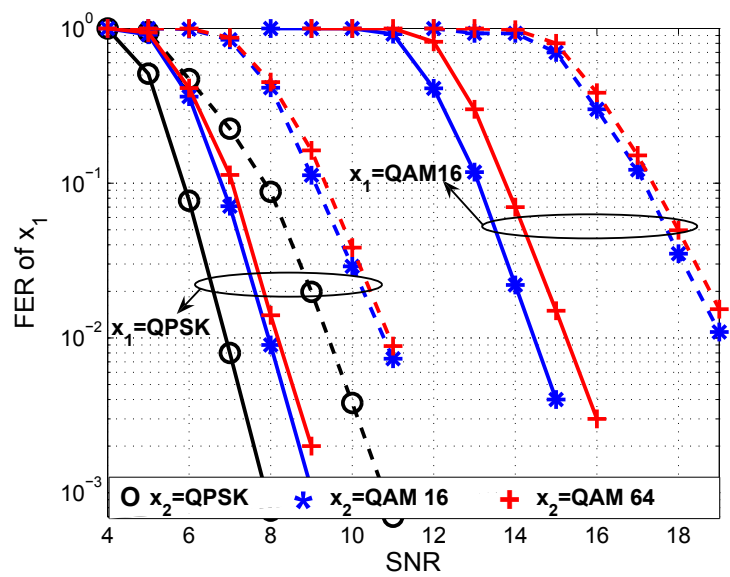

Fig. 8. Performance of lower rate stream in $2 \times 2$ BICM MIMO OFDM system using $802.11 \mathrm{n}$ convolutional code. Continuous lines indicate low complexity max log MAP detector while dashed lines indicate MMSE detector.

\subsection{Simulations}

We consider a $2 \times 2$ BICM MIMO OFDM system using the de facto standard, 64 state rate- $1 / 2$ convolutional encoder of $802.11 \mathrm{n}$ standard $(802.11 \mathrm{n}, 2006)$ and rate- $1 / 2$ punctured turbo code 


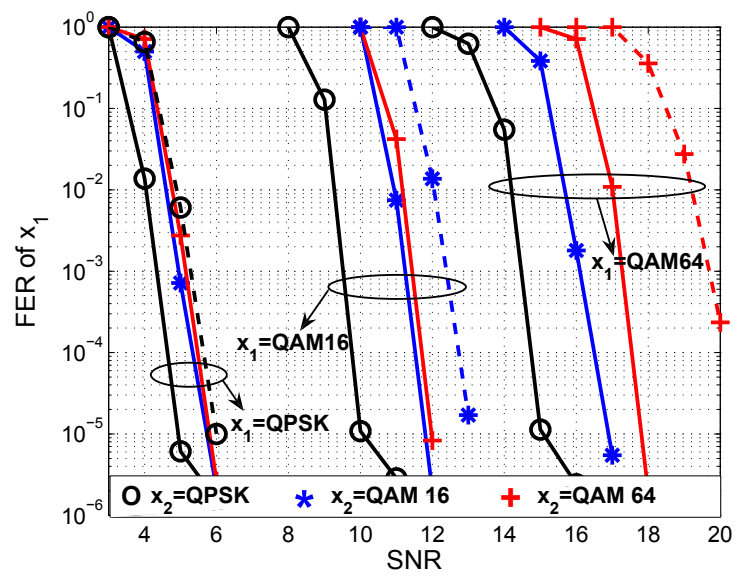

Fig. 9. Performance of lower rate stream in $2 \times 2$ BICM MIMO OFDM system using 3GPP LTE turbo code. Continuous lines indicate low complexity max log MAP detector while dashed lines indicate MMSE detector. Block length of the lower rate stream is 1296 bits while number of decoding iterations are 5 .

proposed for 3GPP LTE (LTE, 2006) ${ }^{1}$. MIMO channel has iid Gaussian matrix entries with unit variance. The channel is independently generated for each time instant and perfect CSI at the receiver is assumed. Furthermore, all mappings of coded bits to QAM symbols use Gray encoding. We consider MMSE and low complexity max log MAP detector. There are two scenarios.

In first scenario, spatial streams of uniform rate and nonuniform power are transmitted in $2 \times$ 2 MIMO broadcast system. The upcoming WLAN standard $802.11 \mathrm{n}(802.11 \mathrm{n}, 2006)$ supports the codeword sizes of 648, 1296, and 1944 bits. For our purposes, we selected the codeword size of 1296 bits and coding scheme of convolutional coding. We focus on the frame error rates (FER) of the system. We consider the low complexity max log MAP and MMSE SIC approach in which the higher power stream is detected first and is subsequently stripped off leading to the detection of lower power stream. With $P_{T}$ being the total power available, the power distribution between two streams is optimized to equate their rates in the desired SNR region where SNR is defined as the received SNR per antenna i.e. $\frac{P_{T}}{N_{0}}$. As a reference, MMSE parallel interference cancellation (PIC) has also been simulated in which two streams are independently detected using MMSE filters and two streams have equal power. Fig. 7 shows the improved performance of low complexity max log MAP approach with respect to both MMSE SIC and MMSE PIC approach. The gap widens as the constellation proliferates i.e QAM 16 and QAM64 which is attributed to the higher suboptimality of MMSE for larger sized constellations.

In second scenario, spatial streams of uniform power and nonuniform rate are transmitted in $2 \times 2$ MIMO broadcast system. We focus on the FER of first stream (lower rate) as subsequent

\footnotetext{
${ }^{1}$ LTE turbo decoder design was performed using the coded modulation library www.iterativesolutions.com
} 
to stripping, the detection of second stream (higher rate) is trivial (using SIMO detectors). The frame length of first stream is fixed to 1296 information bits as per 802.11n (802.11n, 2006). Figs. 8 and 9 compare the performance of low complexity max log MAP detector with MMSE detector. The max log MAP detector performs significantly better than the MMSE detector. Degradation of the performance for first stream as the rate (constellation size) of second stream increases confirms the earlier result of sec. 2.2.2 that rate of first stream is a function of the rate of second stream.

\section{Interference Suppression for future Wireless Systems}

To cope with the ever-increasing demands on the higher spectral efficiency, appendage of spatial dimension (MIMO) needs to be coupled with a tight frequency reuse as is advocated in the future wireless communication systems as 3GPP LTE (LTE, 2006) and LTE-Advanced (LTE-A, 2008). Adaptive modulation and coding schemes will be supported in the next generation wireless systems which combined with the diversified data services will lead to variable transmission rate streams. These system characteristics will overall lead to an interferencelimited system. Most state-of-the-art wireless systems deal with the interference either by orthogonalizing the communication links in time or frequency (Gesbert et al., 2007) or allow the communication links to share the same degrees of freedom but model the interference as additive Gaussian random process (Russell \& Stuber, 1995). Both of these approaches may be suboptimal as first approach entails an $a$ priori loss of the degrees of freedom in both links, independent of the interference strength while second approach treats the interference as pure noise while it actually carries information and has the structure that can be potentially exploited in mitigating its effect.

3GPP LTE (LTE, 2006) has chosen orthogonal frequency division multiple access (OFDMA) technology for the downlink in order to provide multiple access and eliminate the intracell interference. However frequency reuse factor being 1 will lead to intercell interference impairments among neighboring cells. Intercell interference coordination techniques (Gesbert et al., 2007) are studied to minimize the interference level while spatial interference cancellation filters are the focus of attention to cancel the interferers which will be 1 in most cases (near cell boundaries) and 2 in rare cases (near cell corners). Different spatial interference cancellation techniques involving equalization and subtractive cancellation (Bladsjö et al., 1999) (Debbah et al., 2000) have been proposed in the literature. Amongst them, MMSE linear detectors are being considered as likely candidates for 3GPP LTE (Dahlman et al., 2006). The suboptimality of MMSE for non Gaussian alphabets in low dimensional systems (less number of interferers) has already been discussed and simulated in the previous sections and moreover MMSE detection being based on interference attenuation is void of exploiting the interference structure in mitigating its effect. Though not optimal, but their low complexity still makes them attractive for practical systems.

Optimal strategy for treating the interference in the regime of very strong (Carleial, 1975) and very weak interference is well known however if the interference is in the moderate region, no optimal strategy is known but partial decoding of interference can significantly improve performance (Han \& Kobayashi, 1981). This part of the chapter discusses a low complexity spatial interference cancellation algorithm for single frequency reuse synchronized cellular networks in the presence of one strong interferer. This algorithm is based on the low complexity max $\log$ MAP detector and benefits from its ability to exploit interference structure in mitigating its effect. The algorithm encompasses two strategies for interference mitigation i.e. interference suppression and interference cancellation and their selection in the receiver is dictated 


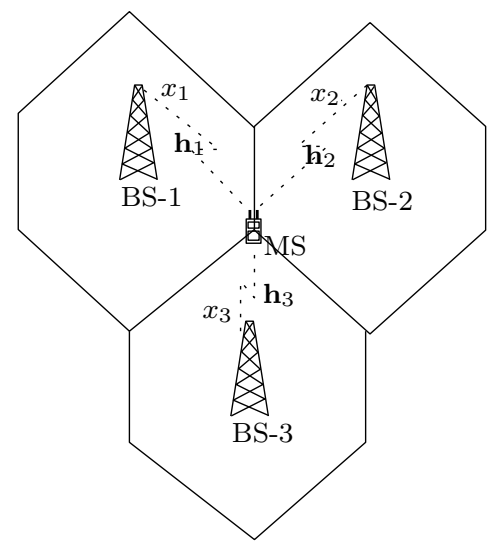

Fig. 10. Interference cancellation in single frequency cellular network. $x_{1}$ is the desired signal while $x_{2}$ and $x_{3}$ are the interference signals.

by the relative strength and the rate of interfering stream. In the scenario of interfering stream being weak or of higher rate relative to the desired stream, thereby making it unfeasible to be decoded, the mobile station (MS) resorts to the strategy of interference suppression. It can be interpreted as partial decoding of the interference which is the recommended strategy in the regime of moderate interference (Han \& Kobayashi, 1981). When the interfering stream is relatively stronger or is of lower rate thereby making it feasible to be decoded, MS adopts interference cancellation strategy (subtractive cancellation) which is the optimal strategy in the case of strong interference (Carleial, 1975).

\subsection{System Model}

The system model as shown in fig. 10 remains same as described in the sec.2.1.2 with 3 spatial streams. However these streams arriving at the receiver (MS) are now from three different base stations (BS) thereby ensuring independent channels. The MS has receive diversity with $n_{r}$ receive antennas. All the BSs are assumed to be synchronous.

\subsection{Information Theoretic view}

For better understanding of the effect of strength and rate (alphabet size) of interference, the case of one strong interference is considered in this section. The focus is on the mutual information of the desired stream in the presence of one strong interferer (Ghaffar \& Knopp, 2009b).

$$
I\left(\mathbf{y} ; x_{1}\right)=\log M_{1}-\frac{1}{M_{1}} \sum_{x_{1}} \int_{\mathbf{y}} p\left(\mathbf{y} \mid x_{1}\right) \log \frac{\sum_{x_{1}} p\left(\mathbf{y} \mid x_{1}\right)}{p\left(\mathbf{y} \mid x_{1}\right)} d \mathbf{y}
$$

Fig. 11 shows the mutual information of the desired stream in the presence of the interference stream. We define the term $\alpha=\sigma_{2}^{2} / \sigma_{1}^{2}$. Mutual information of the desired stream is a function of the rate as well as the strength of the interference stream. For moderate values of $\alpha$ and when the interference has a lower rate (smaller constellation size) relative to the desired stream, as the interference strength increases, the mutual information of the desired stream 


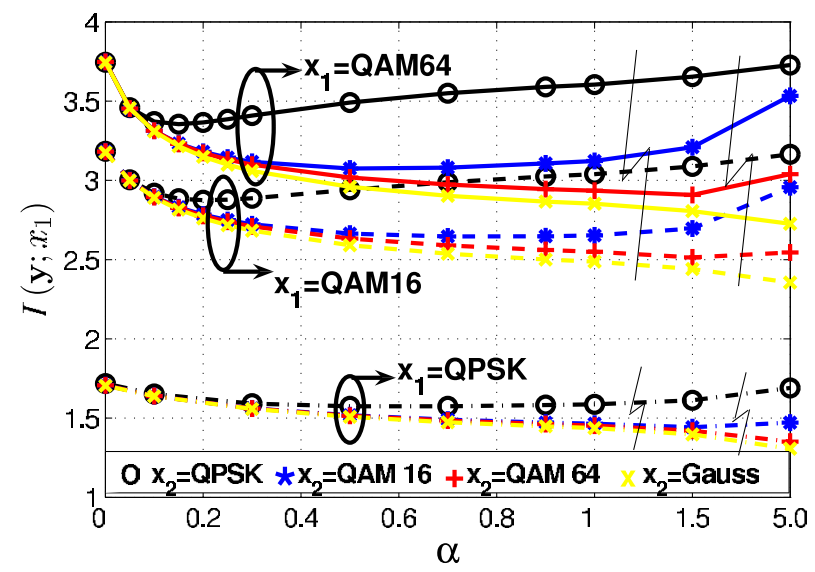

Fig. 11. Mutual information of the desired stream $x_{1}$ in the presence of the interference stream $x_{2}$ for different constellations. SNR is $4.5 \mathrm{~dB}$ for $x_{1}=\mathrm{QPSK}, 11 \mathrm{~dB}$ for $x_{1}=\mathrm{QAM} 16$ and $13 \mathrm{~dB}$ for $x_{1}=\mathrm{QAM} 64$. Note that the flash sign indicates a discontinuity of abscissa.

increases. However when the interference stream has a higher rate as compared to the rate of the desired stream, this behavior is observed for higher values of $\alpha$. This can be interpreted as the decoding capability of the MS of the interference in the presence of the desired stream. Once the interference strength and its rate relative to the strength and the rate of the desired stream permits the decoding of the interference, we observe an increase in the mutual information of the desired stream with the increase of $\alpha$. Fig. 11 also authenticates the well known result of Gaussian being the worst case interference however the gap decreases as the rate of the interference stream increases. This diminution of gap may be related to the proximity of the behavior of large size constellations to Gaussianity as both are characterized by high peak to average power ratios.

\subsection{Interference Mitigation Strategies}

Based on the low complexity max log MAP detector, an interference mitigation strategy (Ghaffar \& Knopp, 2009a) is discussed which is based on the partial decoding of the interference in the regime when interference because of its relative rate or strength is undecodable and subtractive cancellation when the interference is quite strong and is decodable. This strategy is based on exploiting the structure of the interference in mitigating its effect once subtractive cancellation is not possible and resorting to subtractive cancellation otherwise. So there are two options for interference mitigation.

1. In the regime when interference has higher rate or is weaker in strength relative to the desired stream thereby rendering the absolute decoding of interference unfeasible, target stream is decoded using the low complexity max log MAP detector which takes into account the effect of interference and can be termed as the partial decoding of interference or partial joint decoding. This approach is termed as interference suppression.

2. In the regime when interference has lower rate or is stronger in strength relative to the desired stream thereby rendering the absolute decoding of the interference feasible, the 
interference stream is decoded using low complexity max log MAP detector, stripping it off and then decoding the desired stream. This approach is termed as interference cancellation.

The factors that will decide the strategy to be adopted will be the relative rate and the strength of the interference stream comparative to the desired stream. The requisites for this algorithm are the knowledge of interference channel and the modulation and coding scheme (MCS) of interfering stream. The BSs need to be synchronous with pilot signals from the adjacent BSs to be orthogonal to meet these requisites.

\subsection{Performance Analysis}

This section deliberates on the performance analysis of two detectors for detecting the desired stream in the presence of interfering stream (Ghaffar \& Knopp, 2009c).

\subsubsection{PEP Analysis - Max Log MAP Detector}

The conditional PEP i.e. $P\left(\underline{\mathbf{c}}_{1} \rightarrow \underline{\hat{\mathbf{c}}}_{1} \mid \mathbf{H}\right)$ of max log MAP detector is given as

$$
\begin{aligned}
P\left(\underline{\mathbf{c}}_{1} \rightarrow \hat{\mathbf{s}}_{1} \mid \mathbf{H}\right)= & P\left(\sum_{k^{\prime}} \min _{x_{1} \in \chi_{1, c_{k^{\prime}}}^{i}, x_{2} \in \chi_{2}} \frac{1}{N_{0}}\left\|\mathbf{y}_{k}-\mathbf{h}_{1, k} x_{1}-\mathbf{h}_{2, k} x_{2}\right\|^{2} \geq\right. \\
& \left.\sum_{k^{\prime}} \min _{x_{1} \in \chi_{1, \hat{c}_{k^{\prime}}^{\prime}, x_{2} \in \chi_{2}}^{i}} \frac{1}{N_{0}}\left\|\mathbf{y}_{k}-\mathbf{h}_{1, k} x_{1}-\mathbf{h}_{2, k} x_{2}\right\|^{2}\right)
\end{aligned}
$$

where $\mathbf{H}=\left[\mathbf{H}_{1} \cdots \mathbf{H}_{K}\right]$ i.e. the complete channel for the transmission of the codeword $\underline{\mathbf{c}}_{1}$ and $\mathbf{H}_{k}=\left[\mathbf{h}_{1, k} \mathbf{h}_{2, k}\right]$ i.e. the channel at $k$-th frequency tone. For the worst case scenario once $d\left(\underline{\mathbf{c}}_{1}-\underline{\mathbf{s}}_{1}\right)=d_{\text {free }}$, the inequality on the right hand side of (26) shares the same terms on all but $d_{\text {free }}$ summation points for which $\hat{c}_{k^{\prime}}=\bar{c}_{k^{\prime}}$ where $(\overline{.})$ denotes the binary complement. Let

$$
\begin{aligned}
& \tilde{x}_{1, k}, \tilde{x}_{2, k}=\arg \min _{x_{1} \in \chi_{1, c_{k^{\prime}}}^{i}, x_{2} \in \chi_{2}} \frac{1}{N_{0}}\left\|\mathbf{y}_{k}-\mathbf{h}_{1, k} x_{1}-\mathbf{h}_{2, k} x_{2}\right\|^{2} \\
& \hat{x}_{1, k}, \hat{x}_{2, k}=\arg \min _{x_{1} \in \chi_{1, c_{k^{\prime}}}^{i}, x_{2} \in \chi_{2}} \frac{1}{N_{0}}\left\|\mathbf{y}_{k}-\mathbf{h}_{1, k} x_{1}-\mathbf{h}_{2, k} x_{2}\right\|^{2}
\end{aligned}
$$

As $x_{1, k}$ and $x_{2, k}$ are the transmitted symbols so $\left\|\mathbf{y}_{k}-\mathbf{h}_{1, k} x_{1, k}-\mathbf{h}_{2, k} x_{2, k}\right\|^{2} \geq$ $\left\|\mathbf{y}_{k}-\mathbf{h}_{1, k} \tilde{x}_{1, k}-\mathbf{h}_{2, k} \tilde{x}_{2, k}\right\|^{2}$. The conditional PEP is given as

$$
\begin{aligned}
P\left(\underline{\mathbf{c}}_{1} \rightarrow \underline{\mathbf{c}}_{1} \mid \mathbf{H}\right) & \leq P\left(\sum_{k, d_{\text {free }}} \frac{1}{N_{0}}\left\|\mathbf{y}_{k}-\mathbf{h}_{1, k} x_{1, k}-\mathbf{h}_{2, k} x_{2, k}\right\|^{2} \geq \sum_{k, d_{\text {free }}} \frac{1}{N_{0}}\left\|\mathbf{y}_{k}-\mathbf{h}_{1, k} \hat{x}_{1, k}-\mathbf{h}_{2, k} \hat{x}_{2, k}\right\|^{2}\right) \\
& =Q\left(\sqrt{\sum_{k, d_{\text {free }}} \frac{1}{2 N_{0}}\left\|\mathbf{H}_{k}\left(\hat{\mathbf{x}}_{k}-\mathbf{x}_{k}\right)\right\|^{2}}\right) \\
& =Q\left(\sqrt{\frac{1}{2 N_{0}} \operatorname{vec}\left(\overline{\mathbf{H}}^{\dagger}\right)^{\dagger} \Delta \operatorname{vec}\left(\overline{\mathbf{H}}^{\dagger}\right)}\right)
\end{aligned}
$$


where $\overline{\mathbf{H}}=\left[\mathbf{H}_{1} \cdots \mathbf{H}_{k, d_{\text {free }}}\right], \quad \hat{\mathbf{x}}_{k}=\left[\hat{x}_{1, k} \hat{x}_{2, k}\right]^{T}$ and $\boldsymbol{\Delta}=\mathbf{I}_{n_{r}} \otimes \mathbf{D D}^{\dagger}$ while $\mathbf{D}=\operatorname{diag}\left\{\hat{\mathbf{x}}_{1}-\mathbf{x}_{1}, \hat{\mathbf{x}}_{2}-\mathbf{x}_{2}, \cdots, \hat{\mathbf{x}}_{k, d \text { free }}-\mathbf{x}_{k, d_{\text {free }}}\right\} \cdot Q$ is the Gaussian Q-function i.e. $Q(y)=$ $\frac{1}{\sqrt{2 \pi}} \int_{y}^{\infty} e^{-x^{2} / 2} d x$ and vec indicates vectorization of a matrix. For a Hermitian quadratic form in complex Gaussian random variable $q=\mathbf{m}^{\dagger} \mathbf{A m}$ where $\mathbf{A}$ is a Hermitian matrix and column vector $\mathbf{m}$ is a circularly symmetric complex Gaussian vector i.e. $\mathbf{m} \sim \mathcal{N C}(\boldsymbol{\mu}, \boldsymbol{\Sigma})$ with $\boldsymbol{\mu}=E[\mathbf{m}]$ and $\boldsymbol{\Sigma}=E\left[\mathbf{m m}^{\dagger}\right]-\boldsymbol{\mu} \boldsymbol{\mu}^{\dagger}$, the moment generating function (MGF) is

$$
E\left[\exp \left(-t \mathbf{m}^{\dagger} \mathbf{A m}\right)\right]=\frac{\exp \left[-t \boldsymbol{\mu}^{\dagger} \mathbf{A}(\mathbf{I}+t \boldsymbol{\Sigma} \mathbf{A})^{-1} \boldsymbol{\mu}\right]}{\operatorname{det}(\mathbf{I}+t \boldsymbol{\Sigma} \mathbf{A})}
$$

Using Chernoff bound $Q(x) \leq \frac{1}{2} \exp \left(\frac{-x^{2}}{2}\right)$ and the MGF, PEP is upper bounded as

$$
\begin{aligned}
P\left(\underline{\mathbf{c}}_{1} \rightarrow \underline{\hat{\mathbf{c}}}_{1}\right) & \leq \frac{1}{2 \operatorname{det}\left(\mathbf{I}+\frac{1}{4 N_{0}} \mathbf{I} \boldsymbol{\Delta}\right)} \\
& =\frac{1}{2 \prod_{k=1}^{d_{\text {free }}}\left(1+\frac{1}{4 N_{0}}\left\|\hat{\mathbf{x}}_{k}-\mathbf{x}_{k}\right\|^{2}\right)^{n_{r}}}
\end{aligned}
$$

$\left\|\hat{\mathbf{x}}_{k}-\mathbf{x}_{k}\right\|^{2} \geq d_{1, \min }^{2}+d_{2, \min }^{2}$ if $\hat{x}_{2, k} \neq x_{2, k}$ and $\left\|\hat{\mathbf{x}}_{k}-\mathbf{x}_{k}\right\|^{2} \geq d_{1, \min }^{2}$ if $\hat{x}_{2, k}=x_{2, k}$. There exists $2^{d_{\text {free }}}$ possible vectors of $\left[\hat{x}_{2,1}, \cdots, \hat{x}_{2, d_{\text {free }}}\right]^{T}$ basing on the binary criteria that $\hat{x}_{2, k}$ is equal or not equal to $x_{2, k}$. Taking into account all these cases combined with their corresponding probabilities, the PEP is upper bounded as

$P\left(\underline{\mathbf{c}}_{1} \rightarrow \underline{\mathbf{c}}_{1} \mid \mathbf{H}\right) \leq \frac{1}{2}\left(\frac{4 N_{0}}{\sigma_{1}^{2} \breve{d}_{1, \min }^{2}}\right)^{n_{r} d_{\text {free }}}\left(\sum_{j=0}^{d_{\text {free }}} C_{j}^{d_{\text {free }}} \frac{\left(P\left(\hat{x}_{2, k} \neq x_{2, k}\right)\right)^{j}\left(1-P\left(\hat{x}_{2, k} \neq x_{2, k}\right)\right)^{d_{\text {free }}-j}}{\left(1+\frac{\sigma_{2}^{2} \breve{d}_{2, \text { min }}^{2}}{\sigma_{1}^{2} \check{d}_{1, \text { min }}^{2}}\right)^{j n_{r}}}\right)$

where $d_{j, \min }^{2}=\sigma_{j}^{2} \breve{d}_{j, \text { min }}^{2}$ with $\breve{d}_{j, \text { min }}^{2}$ being the normalized minimum distance of the constellation $\chi_{j}$ for $j=\{1,2\}$ and $C_{j}^{d_{\text {free }}}$ is the binomial coefficient. $P\left(\hat{x}_{2, k} \neq x_{2, k}\right)$ has been derived in the following section.

3.4.2 $P\left(\hat{x}_{2, k} \neq x_{2, k}\right)$

Considering (27), $P\left(\hat{x}_{2, k} \neq x_{2, k} \mid \mathbf{h}_{1, k}, \mathbf{h}_{2, k}, x_{1, k}\right)$ is

$$
\begin{aligned}
P\left(\hat{x}_{2, k}\right. & \left.\neq x_{2, k} \mid \mathbf{h}_{1, k}, \mathbf{h}_{2, k}, x_{1, k}\right) \\
& =P\left(-2 \Re\left(\left(\mathbf{h}_{1, k}\left(x_{1, k}-x_{1}\right)+\mathbf{z}_{k}\right)^{\dagger} \mathbf{h}_{2, k}\left(x_{2, k}-x_{2}\right)\right) \geq\left\|\mathbf{h}_{2, k}\left(x_{2, k}-x_{2}\right)\right\|^{2} \mid \mathbf{H}_{k}, x_{1, k}\right) \\
& =Q\left(\sqrt{\frac{\left\|\mathbf{h}_{2, k}\left(x_{2, k}-x_{2}\right)\right\|^{2}}{2 N_{0}}}+\sqrt{\frac{2}{N_{0}}} \Re\left(\frac{\left(\mathbf{h}_{1, k}\left(x_{1, k}-x_{1}\right)\right)^{\dagger} \mathbf{h}_{2, k}\left(x_{2, k}-x_{2}\right)}{\sqrt{\left\|\mathbf{h}_{2, k}\left(x_{2, k}-x_{2}\right)\right\|^{2}}}\right)\right)
\end{aligned}
$$


Using the relation $Q(a+b) \leq Q\left(a_{\min }-\left|b_{\max }\right|\right)$ and $\Re\left(\mathbf{a}^{+} \hat{\mathbf{b}}\right) \leq\|\mathbf{a}\|$ where $\hat{\mathbf{b}}$ is the unit vector we get

$P\left(\hat{x}_{2, k} \neq x_{2, k} \mid \mathbf{h}_{1, k}, \mathbf{h}_{2, k}\right) \leq \frac{1}{2} \exp \left(-\frac{\left\|\mathbf{h}_{2, k}\right\|^{2} d_{2, \min }^{2}}{4 N_{0}}-\frac{\left\|\mathbf{h}_{1, k}\right\|^{2} d_{1, \max }^{2}}{N_{0}}+\frac{\left\|\mathbf{h}_{2, k}\right\|\left\|\mathbf{h}_{1, k}\right\| d_{2, \min } d_{1, \max }}{N_{0}}\right)$

Conditioned on the norm of $\mathbf{h}_{1, k}$ we make two non-overlapping regions as $\left(\left\|\mathbf{h}_{2, k}\right\| \geq\left\|\mathbf{h}_{1, k}\right\| \mid \mathbf{h}_{1, k}\right)$ and $\left(\left\|\mathbf{h}_{2, k}\right\|<\left\|\mathbf{h}_{1, k}\right\| \mid \mathbf{h}_{1, k}\right)$ with the corresponding probabilities as $\mathcal{P}_{\mathbf{h}_{1}}^{<}$and $\mathcal{P}_{\mathbf{h}_{1}}^{>}$. Note that in first region $\left\|\mathbf{h}_{2, k}\right\|\left\|\mathbf{h}_{1, k}\right\| \leq\left\|\mathbf{h}_{2, k}\right\|^{2}$ while for second region $\left\|\mathbf{h}_{2, k}\right\|\left\|\mathbf{h}_{1, k}\right\|<\left\|\mathbf{h}_{1, k}\right\|^{2}$. So

$$
\begin{aligned}
& P\left(\hat{x}_{2, k} \neq x_{2, k}\right) \leq \frac{1}{2} E_{\mathbf{h}_{1}}\left[\left(\frac{4 N_{0}}{d_{2, \text { min }}^{2}-4 d_{2, \min } d_{1, \max }}\right)^{n_{r}} \exp \left(-\frac{\left\|\mathbf{h}_{1, k}\right\|^{2} d_{1, \max }^{2}}{N_{0}}\right) E_{\mathbf{h}_{2} \mid \mathbf{h}_{1}}\left(\mathcal{P}_{\mathbf{h}_{1}}^{<}\right)\right. \\
& \left.+\left(\frac{4 N_{0}}{d_{2, \min }^{2}}\right)^{n_{r}} \exp \left(-\left\|\mathbf{h}_{1, k}\right\|^{2} \frac{d_{1, \max }^{2}-d_{2, \min } d_{1, \max }}{N_{0}}\right) E_{\mathbf{h}_{2} \mid \mathbf{h}_{1}}\left(\mathcal{P}_{\mathbf{h}_{1}}^{>}\right)\right] \\
& \leq \frac{1}{2}\left(\frac{4 N_{0}}{\sigma_{2}^{2} \breve{d}_{2, \text { min }}^{2}}\right)^{n_{r}}\left(\frac{N_{0}}{\sigma_{1}^{2} \breve{d}_{1, \text { max }}^{2}}\right)^{n_{r}}\left(\frac{1}{\left(1-\frac{4 \sigma_{1} \breve{d}_{1, \text { max }}}{\sigma_{2} \breve{d}_{2, \text { min }}}\right)^{n_{r}}}+\frac{1}{\left(1-\frac{\sigma_{2} \breve{d}_{2, \text { min }}}{\sigma_{1} \breve{d}_{1, \text { max }}}\right)^{n_{r}}}\right)
\end{aligned}
$$

where we upper bound $E_{\mathbf{h}_{2} \mid \mathbf{h}_{1}}\left(\mathcal{P}_{\mathbf{h}_{1}}^{<}\right)$and $E_{\mathbf{h}_{2} \mid \mathbf{h}_{1}}\left(\mathcal{P}_{\mathbf{h}_{1}}^{>}\right)$by 1 .

$P\left(\hat{x}_{2, k} \neq x_{2, k}\right) \rightarrow 0$ as $\sigma_{2}^{2} \rightarrow \infty$ while $P\left(\hat{x}_{2, k} \neq x_{2, k}\right)$ increases as $\sigma_{2}^{2}$ increases. Eq. (31) demonstrates a significant result of achieving full diversity by the low complexity max log MAP detector and converging to the performance of single stream using maximum ratio combining in the case of very weak and strong interference. In the moderate region, as the strength of interference increases, $P\left(\hat{x}_{2, k} \neq x_{2, k}\right)$ reduces and there is a coding gain for the detection of desired stream contrary to the case of MMSE where there is a coding loss as the interference gets stronger (shown in the next section).

\subsubsection{PEP Analysis - MMSE Detector}

\subsection{Gaussian Assumption}

Conditional PEP for MMSE basing on Gaussian assumption of post detection interference (20) is given as

$$
P\left(\underline{\mathbf{c}}_{1} \rightarrow \underline{\mathbf{c}}_{1} \mid \mathbf{H}\right)=P\left(\sum_{k^{\prime}} \min _{x_{1} \in \chi_{1, c_{k^{\prime}}}^{i}} \frac{\left|y_{k}-\alpha_{k} x_{1}\right|^{2}}{N_{k}} \geq \sum_{k^{\prime}} \min _{x_{1} \in \chi_{1, \hat{c}_{k^{\prime}}}^{i}} \frac{\left|y_{k}-\alpha_{k} x_{1}\right|^{2}}{N_{k}}\right)
$$

Let

$$
\tilde{x}_{1, k}=\arg \min _{x_{1} \in \chi_{1, c_{k^{\prime}}}^{i}} \frac{\left|y_{k}-\alpha_{k} x_{1}\right|^{2}}{N_{k}}, \quad \hat{x}_{1, k}=\arg \min _{x_{1} \in \chi_{1, \tilde{c}_{k^{\prime}}}^{i}} \frac{\left|y_{k}-\alpha_{k} x_{1}\right|^{2}}{N_{k}}
$$


Considering the worst case scenario $d\left(\underline{\mathbf{c}}_{1}-\underline{\hat{\mathbf{c}}}_{1}\right)=d_{\text {free }}$ and using the fact that $\frac{1}{N_{k}}\left|y_{k}-\alpha_{k} x_{1, k}\right|^{2} \geq \frac{1}{N_{k}}\left|y_{k}-\alpha_{k} \tilde{x}_{1, k}\right|^{2}$, the conditional PEP is upper bounded as

$$
P\left(\underline{\mathbf{c}}_{1} \rightarrow \underline{\mathbf{c}}_{1} \mid \mathbf{H}\right) \leq Q\left(\sqrt{\sum_{k, d_{\text {free }}} \frac{\alpha_{k}^{2}}{2 N_{k}}\left|\hat{x}_{1, k}-x_{1, k}\right|^{2}}\right)
$$

Bounding $\left|\hat{x}_{1, k}-x_{1, k}\right|^{2} \geq d_{1, \min }^{2}$ and using the Chernoff bound

$$
P\left(\underline{\mathbf{c}}_{1} \rightarrow \underline{\hat{\mathbf{c}}}_{1} \mid \mathbf{H}\right) \leq \frac{1}{2} \exp \left(-\frac{d_{1, \min }^{2}}{4} \sum_{k, d_{\text {free }}} \mathbf{h}_{1, k}^{\dagger} \mathbf{R}_{2, k}^{-1} \mathbf{h}_{1, k}\right)
$$

where the summation in (36) can be written as

$$
\sum_{k, d_{\text {free }}} \mathbf{h}_{1, k}^{\dagger} \mathbf{R}_{2, k}^{-1} \mathbf{h}_{1, k}=\left[\mathbf{h}_{1,1}^{\dagger}, \cdots, \mathbf{h}_{1, d_{\text {free }}}^{\dagger}\right] \operatorname{diag}\left[\mathbf{R}_{2,1}^{-1}, \cdots, \mathbf{R}_{2, d_{\text {free }}}^{-1}\right]\left[\mathbf{h}_{1,1}^{T}, \cdots, \mathbf{h}_{1, d_{\text {free }}}^{T}\right]^{T}
$$

The eigenvalues of $\mathbf{R}_{2, k}^{-1}$ are

$$
\lambda_{l}= \begin{cases}\left(\sigma_{2}^{2}\left\|\mathbf{h}_{2, k}\right\|^{2}+N_{0}\right)^{-1}, & l=1 \\ N_{0}^{-1}, & l=2, \cdots, n_{r}\end{cases}
$$

Using the MGF (29), PEP conditioned on $\overline{\mathbf{h}}_{2}=\left[\mathbf{h}_{2,1}, \cdots, \mathbf{h}_{2, d_{\text {free }}}\right]$ is upper bounded as

$$
P\left(\underline{\mathbf{c}}_{1} \rightarrow \underline{\hat{\mathbf{c}}}_{1} \mid \overline{\mathbf{h}}_{2}\right) \leq \frac{1}{2}\left(\frac{4 N_{0}}{d_{1, \min }^{2}}\right)^{d_{\text {free }}\left(n_{r}-1\right)}\left(\frac{4}{d_{1, \min }^{2}}\right)^{d_{\text {free }}} \prod_{l=1}^{d_{\text {free }}}\left(\sigma_{2}^{2}\left\|\mathbf{h}_{2, l}\right\|^{2}+N_{0}\right)
$$

Channel independence at each subcarrier yields

$$
P\left(\underline{\mathbf{c}}_{1} \rightarrow \underline{\hat{\mathbf{c}}}_{1}\right) \leq \frac{1}{2}\left(\frac{4 N_{0}}{\sigma_{1}^{2} \breve{d}_{1, \min }^{2}}\right)^{d_{\text {free }}\left(n_{r}-1\right)}\left(\frac{4}{\sigma_{1}^{2} \breve{d}_{1, \text { min }}^{2}}\right)^{d_{\text {free }}}\left(n_{r} \sigma_{2}^{2}+N_{0}\right)^{d_{\text {free }}}
$$

which not only demonstrates the well known result of the loss of one diversity order in MMSE in the presence of one interferer (Winters, 1984) but also exhibits a coding loss as interference gets stronger.

\subsection{Simulation Results}

Moderate and high SNR regime in the interference-limited scenario demands more attention as when the noise is small, interference will have a significant impact on the performance. Low SNR regime is less interesting since here the performance is noise-limited and interference is not having a significant effect. For simulations, we have restricted ourselves to the case of one strong interference. These simulations have been performed in moderate and high SNR region while the interference strength is being varied.

We consider 2 BSs each using BICM OFDM system for downlink transmission using the de facto standard, 64 state $(133,171)$ rate- $1 / 2$ convolutional encoder of $802.11 n$ standard $(802.11 n$, 


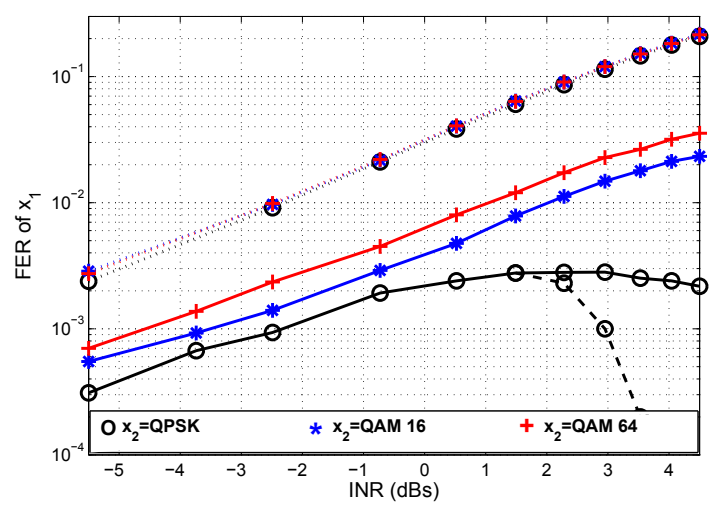

Fig. 12. Desired stream $x_{1}$ is QPSK while interference stream $x_{2}$ is from QPSK, QAM16 and QAM64. SNR is $4.5 \mathrm{~dB}$. Continuous lines indicate interference suppression while dashed lines indicate interference cancellation. Dotted lines indicates detection of $x_{1}$ by MMSE detector. $64-$ state, rate 1/2 Convolutional Code is used. Note that SNR is with respect to the desired stream

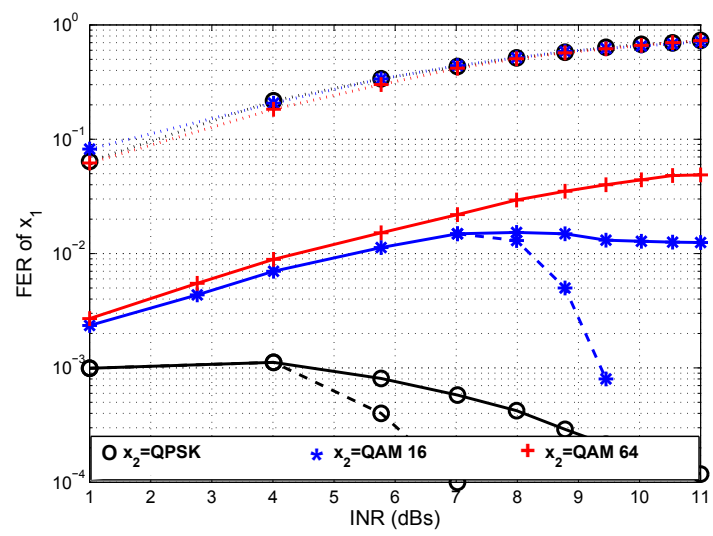

Fig. 13. Desired stream $x_{1}$ is QAM 16 while interference stream $x_{2}$ is from QPSK, QAM16 and QAM64. SNR is $11 \mathrm{~dB}$. Continuous lines indicate interference suppression while dashed lines indicate interference cancellation. Dotted lines indicates detection of $x_{1}$ by MMSE detector. $64-$ state, rate $1 / 2$ Convolutional Code is used.

2006) and the punctured rate $1 / 2$ turbo code of 3GPP LTE (LTE, 2006) $)^{2}$. Each BS has multiple antennas and employs antenna cycling. MS has two antennas. We consider an ideal OFDM

${ }^{2}$ The LTE turbo decoder design was performed using the coded modulation library www.iterativesolutions.com 


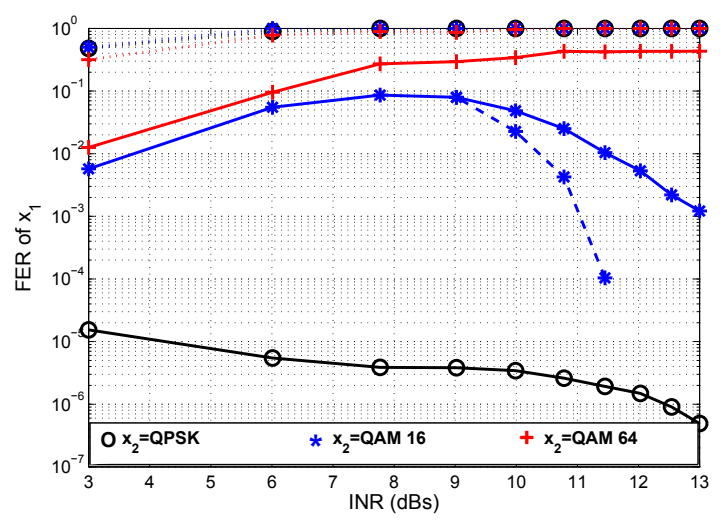

Fig. 14. Desired stream $x_{1}$ is QAM 64 while interference stream $x_{2}$ is from QPSK, QAM16 and QAM64. SNR is $13 \mathrm{~dB}$. Continuous lines indicate interference suppression while dashed lines indicate interference cancellation. Dotted lines indicates detection of $x_{1}$ by MMSE detector. Punctured rate 1/2 3GPP turbo code is used with 5 decoding iterations.

based system (no ISI) and analyze the system in frequency domain. Due to bit interleaving followed by OFDM, this can be termed as frequency interleaving. Therefore SIMO channel at each sub carrier from BS to MS has iid Gaussian matrix entries with unit variance. Perfect CSI is assumed at the receiver. Furthermore, all mappings of coded bits to QAM symbols use Gray encoding. We consider interference suppression and interference cancellation approaches using low complexity max log MAP detector. For comparison we also consider interference suppression using MMSE detector.

Figs. 12, 13 and 14 show the FERs of target stream in the presence of one interference stream. These simulation results show that the dependence of the performance for MMSE detection is insignificant on the rate of the interference stream but its dependence on interference strength is substantial. This can be interpreted as a consequence of the attenuation of interference strength at the output of MMSE filter and the subsequent assumption of Gaussianity for its behavior. For the low complexity max log MAP detector, a significant improvement is observed in the performance as the rate of interference stream decreases which is in conformity with the earlier results of mutual information analysis (fig. 11). It is observed that for a given interference level, the performance is generally degraded as the rate (constellation size) of the interfering stream increases. The performance gap with respect to MMSE decreases as the desired and the interference streams grow in constellation size which can be attributed to the proximity to the Gaussianity of these larger constellations due to their high peak to average power ratio and to the optimality of MMSE for Gaussian alphabets.

\section{References}

802.11n (2006). Enhancements for Higher Throughput, IEEE 802.11 WG. IEEE 802.11n/D1.0 Draft Amendment.

802.16m (2007). Draft IEEE 802.16m Evaluation Methodology, IEEE 802.16m-07/037r1. 
Alamouti, S. (1998). A simple transmit diversity technique for wireless communications, IEEE Journal on Selected Areas in Communications Vol. 16(No. 8): 1451-1458.

Bladsjö, D., Furuskär, A., Jäverbring, S. \& Larsson, E. (1999). Interference cancellation using antenna diversity for EDGE - Enhanced data rates in GSM and TDMA/136, IEEE Vehicular Technology Conference Proceedings, 1999. VTC 1999-Fall, Vol. 4, pp. 1956-1960.

Caire, G., Taricco, G. \& Biglieri, E. (1998). Bit-interleaved coded modulation, Vol. 44(No. 3): 927-946.

Carleial, A. (1975). A case where Interference does not reduce Capacity, IEEE Transactions on Information Theory Vol. 21(No. 5): 569-570.

Dahlman, E., Ekstrom, H., Furuskar, A., Jading, Y., Karlsson, J., Lundevall, M. \& Parkvall, S. (2006). The $3 \mathrm{G}$ long-term evolution - radio interface concepts and performance evaluation, IEEE 63rd Vehicular Technology Conference VTC-Spring, Vol. 1, pp. 137-141.

Debbah, M., Muquet, B., de Courville, M., Muck, M., Simoens, S. \& Loubaton, P. (2000). A MMSE successive interference cancellation scheme for a new adjustable hybrid spread OFDM system, IEEE 51st Vehicular Technology Conference Proceedings, 2000. VTC 2000-Spring Tokyo., Vol. 2, pp. 745-749 vol.2.

Foschini, G. J. \& Gans, M. J. (1998). On limits of wireless communication in a fading environment when using multiple antennas, Wireless Personal Communications. Vol. 6(No. 3): 311-335.

Gesbert, D., Kiani, S., Gjendemsj, A. \& Oien, G. (2007). Adaptation, coordination, and distributed resource allocation in interference-limited wireless networks, Proceedings of the IEEE Vol. 95(No. 12): 2393-2409.

Ghaffar, R. \& Knopp, R. (2008a). Dual-antenna BICM reception with applications to MIMO broadcast and single frequency cellular system, IEEE 19-th International Symposium on Personal, Indoor and Mobile Radio Communications (PIMRC 2008), Cannes.

Ghaffar, R. \& Knopp, R. (2008b). Low complexity BICM demodulation for MIMO transmission, 9th IEEE Workshop on Signal Processing Advances for Wireless Communications, SPAWC 2008, Recife.

Ghaffar, R. \& Knopp, R. (2009a). Interference Suppression for Next Generation Wireless Systems, IEEE 69-th Vehicular Technology Conference VTC-Spring 2009, Barcelona.

Ghaffar, R. \& Knopp, R. (2009b). Spatial Interference Cancellation Algorithm, Proc. IEEE Wireless Communications and Networking Conference WCNC 2009, Budapest, Budapest, Hungary.

Ghaffar, R. \& Knopp, R. (2009c). Spatial Interference Cancellation and Pairwise Error Probability Analysis, IEEE International Conference on Communications, ICC 2009.

Golden, G., Foschini, C., Valenzuela, R. \& Wolniansky, P. (1999). Detection algorithm and initial laboratory results using v-blast space-time communication architecture, Electronics Letters Vol. 35(No. 1): 14-16.

Gonzalez-Lopez, M., Vazquez-Araujo, F., Castedo, L. \& Garcia-Frias, J. (2006). Optimized serially-concatenated LDGM and Alamouti codes for approaching MIMO Capacity, IEEE 17th International Symposium on Personal, Indoor and Mobile Radio Communications, PIMRC 2006, pp. 1-5.

Han, T. \& Kobayashi, K. (1981). A new achievable rate region for the interference channel, IEEE Transactions on Information Theory Vol. 27(No. 1): 49-60.

Hochwald, B. \& Brink, S. T. (2003). Achieving near-capacity on a multiple-antenna channel, IEEE Transactions on Communications, Vol. 51(No. 3): 389-399. 
Larsson, E. G. \& Stoica, P. (2003). Space-Time Block Coding for Wireless Communications, Cambridge University Press, Cambridge, U.K.

Larsson, E. \& Jalden, J. (2008). Fixed-complexity soft MIMO detection via partial marginalization, IEEE Transactions on Signal Processing, Vol. 56(No. 8): 3397-3407.

Liu, Y., Lau, K., Takeshita, O. \& Fitz, M. (2002). Optimal rate allocation for superposition coding in quasi-static fading channels, IEEE International Symposium on Information Theory, ISIT 2002, pp. 111-.

LTE (2006). Requirements for Evolved UTRA (E-UTRA) and Evolved UTRAN (E-UTRAN), 3GPP TR 25.913 v.7.3.0,.

LTE-A (2008). Requirements for Further Advancements for EUTRA, 3GPP TR 36.913,.

Medvedev, I., Bjerke, B., Walton, R., Ketchum, J., Wallace, M. \& Howard, S. (2006). A comparison of MIMO receiver structures for 802.11N WLAN - Performance and complexity, IEEE 17th International Symposium on Personal Indoor and Mobile Radio Communications, pp. 1-5.

Poor, H. \& Verdu, S. (1997). Probability of error in MMSE multiuser detection, IEEE Transactions on Information Theory Vol. 43(No. 3): 858-871.

Russell, M. \& Stuber, G. (1995). Interchannel interference analysis of OFDM in a mobile environment, IEEE 45th Vehicular Technology Conference, Vol. 2, pp. 820-824.

Shamai, S. \& Steiner, A. (2003). A broadcast approach for a single-user slowly fading MIMO channel, IEEE Transactions on Information Theory Vol. 49(No. 10): 2617-2635.

Telatar, I. E. (1999). Capacity of multi-antenna Gaussian channels, European Transactions on Telecommunications Vol. 10(No. 6): 585-595.

Varanasi, M. K. \& Guess, T. (1997). Optimum decision feedback multiuser equalization with successive decoding achieves the total capacity of the Gaussian MAC channel, Asilomar Conference on Signals, Systems and Computers.

Verdu, S. (1989). Computational complexity of multiuser detection, Algorithmica,, Vol. 4, pp. 303-312.

Verdu, S. (1998). Multiuser Detection., Cambridge University Press, Cambridge, U.K.

Winters, J. (1984). Optimum combining in digital mobile radio with cochannel interference, IEEE Journal on Selected Areas in Communications Vol. 2(No. 4): 528-539.

Wolniansky, P., Foschini, G., Golden, G. \& Valenzuela, R. (1998). V-blast: an architecture for realizing very high data rates over the rich-scattering wireless channel, International Symposium on Signals, Systems, and Electronics, 1998. ISSSE 98., pp. 295-300. 


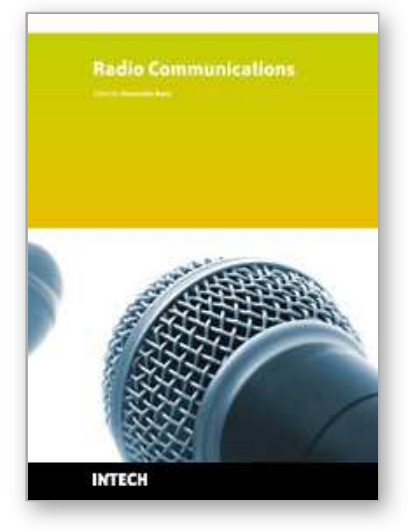

\author{
Radio Communications \\ Edited by Alessandro Bazzi
}

ISBN 978-953-307-091-9

Hard cover, 712 pages

Publisher InTech

Published online 01, April, 2010

Published in print edition April, 2010

In the last decades the restless evolution of information and communication technologies (ICT) brought to a deep transformation of our habits. The growth of the Internet and the advances in hardware and software implementations modified our way to communicate and to share information. In this book, an overview of the major issues faced today by researchers in the field of radio communications is given through 35 high quality chapters written by specialists working in universities and research centers all over the world. Various aspects will be deeply discussed: channel modeling, beamforming, multiple antennas, cooperative networks, opportunistic scheduling, advanced admission control, handover management, systems performance assessment, routing issues in mobility conditions, localization, web security. Advanced techniques for the radio resource management will be discussed both in single and multiple radio technologies; either in infrastructure, mesh or ad hoc networks.

\title{
How to reference
}

In order to correctly reference this scholarly work, feel free to copy and paste the following:

Rizwan Ghaffar and Raymond Knopp (2010). Low Dimensional MIMO Systems with Finite Sized Constellation Inputs, Radio Communications, Alessandro Bazzi (Ed.), ISBN: 978-953-307-091-9, InTech, Available from: http://www.intechopen.com/books/radio-communications/low-dimensional-mimo-systems-with-finite-sizedconstellation-inputs

\section{INTECH}

open science | open minds

\section{InTech Europe}

University Campus STeP Ri

Slavka Krautzeka 83/A

51000 Rijeka, Croatia

Phone: +385 (51) 770447

Fax: +385 (51) 686166

www.intechopen.com

\section{InTech China}

Unit 405, Office Block, Hotel Equatorial Shanghai

No.65, Yan An Road (West), Shanghai, 200040, China 中国上海市延安西路65号上海国际贵都大饭店办公楼 405 单元 Phone: +86-21-62489820

Fax: $+86-21-62489821$ 
(C) 2010 The Author(s). Licensee IntechOpen. This chapter is distributed under the terms of the Creative Commons Attribution-NonCommercialShareAlike-3.0 License, which permits use, distribution and reproduction for non-commercial purposes, provided the original is properly cited and derivative works building on this content are distributed under the same license. 